\title{
On the gradient elastic wave propagation in cylindrical waveguides with microstructure
}

\author{
Antonios Charalambopoulos*, Leonidas N. Gergidis, Giorgos Kartalos \\ Department of Materials Science and Engineering, \\ University of Ioannina, 45110 Ioannina, Greece \\ *email: acharala@uoi.gr \\ Keywords: A. Smart Materials; B. Elasticity; C. Analytical Modelling
}

January 3, 2012

\begin{abstract}
The present work describes the development of a complete theoretical framework of wave propagation in cylindrical waveguides possessing microstructure. In parallel, a thorough investigation of the full 3-D model of wave propagation in cylinders is presented. The first step is the spectral decomposition of the boundary value problem emerging via wave propagation analysis. The spectral representation of the specific gradient elasticity problem reflects the ability to construct all the possible propagating modes in cylindrical geometry. Several byproducts arise along the present work, which constitute generalizations of well known important features of classical elasticity and are indispensable for modeling the gradient elasticity problem. We note the construction of the set of dyadic Navier eigenfunctions which constitute the generalization of the Navier eigenvectors. The restriction of the Navier eigendyadics on cylindrical surfaces gives birth to the dyadic cylindrical harmonics, which constitute the generalization of the well known vector harmonics. This set is also a basis in the sense that the trace of every dyadic field on a cylindrical surface can be represented as a countable superposition of dyadic cylindrical harmonics. The method aims at providing the necessary theoretical establishment for the determination of the dispersion curves emerging in cortical bones.
\end{abstract}

\section{Introduction}

The classical theory of elasticity is insufficient to describe the mechanical behavior of linear elastic materials when these materials exhibit a specific type of microstructure. Liquid crystals, porous media, granular materials, polymers, solids with micro cracks, dislocations and disclinations are illustrative cases where the use of micro continuum field theories is necessary to provide appropriate solutions. 
The necessity of utilization of the so called micro-continuum theories is clarified when we encounter the notion of length and time scale characterizing wave fields participating in physical processes within materials with micro-structure. For example, in [1] the authors investigate the propagation and scattering of elastic plane waves by spherical inclusions with micro-structure, revealing the necessity to develop enhanced elastic theories when the characteristic wave-length $\lambda$ of the exciting incident field is comparable to some internal characteristic length $l$ of the medium. In that case the response of every sub-continuum region to the external stimulus obtains an individual role, rendering unsuccessfull the axiom of locality. As an example, the classical theory of elasticity is well known to predict two non dispersive waves (the longitudinal and the transverse one) whose short wavelength behavior departs essentially from experimental observations. To take into consideration the non locality of the involved elastic fields, several approaches have been followed modeling the microstructural effects in a macroscopic manner by introducing higher-order strain gradient, micropolar and couple stress theories. We note here the principal contributions of Mindlin and co-workers [2],[3],[4], Aifantis and co-workers [5], [6],[7],[8] and Vardoulakis and co-workers [9],[10] in connection with the higher-order strain theories. In addition one may pay attention to the contribution of Eringen and co-workers $[11],[12]$ and [13] pertaining to the micropolar theories, as well as [14], [15], [16] and $[17]$ in connection with the couple-stress theories.

The implication of non classical theories in dynamic problems involving wave propagation in beams and half-space has proved very promising [18],[19],[20] by eliminating singularities or discontinuities of classical theory and capturing the expected size effects and wave dispersion in cases where this was not possible by classical elasticity methods. Especially the propagation of guided elastic waves inside "guiding" elastic structures characterized by co-existence of several spatial scales is a physical problem of great significance from the theoretical and applications point of view. Several multiple scattering phenomena arise generated by the internal micro-components of the structure in conjunction with the physical boundaries of the waveguide. Special study of elastic wave propagation incorporating enhanced theories is attributed to Georgiadis and his co-authors [21], [22].

From the application point of view, the investigation of guided waves in elastic structures mimicking - although via a simple primitive 2 -D model - the cortical bones, have attracted the interest of the authors in [23] and [24], where it has been shown that gradient elasticity can provide supplementary information to better understand guided waves in porous media physically mimicking the bone structure. These works have been suggested as alternative approaches to a series of primitive studies ([25],[26], [27]) aiming at investigating non destructive monitoring of biomedical systems within the framework of classical elastic wave propagation. Thus the new approach revealed the importance of taking into account the inner microstructure of the underlying physical systems.

In this work, there are two motives: the first one is to develop the complete theoretical framework of wave propagation in cylindrical waveguides possessing microstructure and the second one is the thorough investigation of the full 3- 
D model of wave propagation in cylinders mimicking long bones through the use of appropriate geometry and microstructural parameters. In Section 2 we give the connection of gradient elasticity with cortical bone. As far as the first aim is concerned, we construct in Section 3 all the necessary theoretical tools, very reminiscent of the notions introduced in [1], in order to obtain the spectral decomposition of the boundary value problem emerging via wave propagation analysis. The spectral representation of the specific gradient elasticity problem reflects the ability to construct all the possible propagating modes in cylindrical geometry. Several byproducts arise along the present work, which constitute generalizations of well known important features of classical elasticity and are indispensable for modeling the gradient elasticity problem. We note first that we follow the very efficient dyadic formulation, conceived in [28] for practical rather than physical reasons as already revealed in [1]. We focus also on the construction of the set of dyadic Navier eigenfunctions which constitutes the generalization of the Navier eigenvectors. This set is important since every interior or exterior (radiating or attenuated) dyadic field of gradient elasticity can be expressed as a countable expansion in terms of the elements of this set. This fact is a fruitful spectral representation of solutions of the dynamic equation of gradient elasticity in cylindrical coordinates. It is important to note that the restriction of the Navier eigendyadics on cylindrical surfaces gives birth to the dyadic cylindrical harmonics, which constitute the generalization of the well known vector entities. This set is also a basis in the sense that the trace of every dyadic field on a cylindrical surface can be represented as a countable superposition of dyadic cylindrical harmonics.

In Section 4, we exploit the already constructed spectral representation to solve the problem of wave propagation. More precisely, we force this representation to satisfy the free boundary conditions on the surface of the waveguide. The resulting boundary conditions on the cylindrical surface are functionally "projected" on the basis of the dyadic cylindrical harmonics and this process in conjunction with the already constructed -in Section 2- orthogonality properties of the harmonics basis separates the infinite spectral representation and leads to several linear finite algebraic systems. These systems involve as unknowns the wave spectral representation coefficients and are homogeneous due to the free boundary conditions. Thus the determinants of these systems vanish giving birth to the construction of the dispersion relations along with the propagating modes within the waveguide. These dispersion relations turn out to be very complicated functions involving several constitutive parameters, and interrelate the frequency $\omega$ with the axial wave number $p$. In particular, the dependence of the dispersion relation on $p$ is tremendously involved and highly non linear. Thus the application of the present mathematical methodology to the determination of the dispersion curves corresponding to the propagating modes inside long cylinders - simulating cortical bones - requires a thorough multi-parametric analysis w.r.t several physical and geometrical features of the problem. This last task can not be a part of the present work, is independent, consists of a very demanding numerical implementation involving consecutive computer experiments and constitutes the target of work under preparation. 


\section{Gradient elasticity and cortical bone}

In recent years, gradient elastic theory has gained significant interest in deriving analytical and numerical solutions of ultrasound propagation through cortical bone [23], [24]. Although classical elastic theory has been extensively used in bone studies it cannot provide a complete description of dynamic mechanical behavior since it is associated with concepts of homogeneity and locality of stresses. However, experimental observations have shown that in heterogeneous materials with dimensions comparable to the length scale of the microstructure, microstructural effects become important. In addition, the state of stress has to be theoretically defined in a non-local manner.

Bones microstructural effects can be successfully modeled in a macroscopic framework by employing enhanced elastic theories such as the couple stresses theory proposed by Cosserat brothers [14] and generalized later by Eringen as the micropolar elastic theory [40], the general higher-order gradient elastic theory proposed by Mindlin [2], and the non-local theory of elasticity of Eringen [41] .

Furthermore, the versions of Mindlin's general elasticity theory with microstructure (Georgiadis et al.[22], Tsepoura et al.[18]) have gained significant interest since all tensors involved are symmetric being thus mathematically simpler and more understandable from a physical point of view. One of the most crucial and difficult issues in the enhanced theories as they apply to real problems is the determination of the internal length scale parameters. Very recently Vavva et al. [24], exploiting the dipolar gradient elastic theory, derived analytically dispersion curves of guided waves propagating in a two dimensional and free of stress gradient elastic plate mimicking the cortical bone. The two lengthscale parameters introduced in the considered dipolar gradient elastic theory were taken empirically to be about the size of an osteon. It was demonstrated in [24] that when the elastic constants have different values, microstructure plays a significant role in the propagation of the bulk longitudinal and shear waves by inducing material and geometrical dispersion. It was also shown that the insertion of the microstructural characteristics into the stress analysis gives rise to major changes in the dispersion of the guided modes predicted by the classical Lamb wave theory. This work was further extended by Papacharalampopoulos et al. [38] by introducing four microstructural parameters in order to have a more realistic modeling of cortical bone, since different intrinsic parameters for shear and longitudinal waves are necessary. More specifically Pacacharalambopoulos et al. [38] provide in Section IVa a clear and thorough connection between the bone elastic and material parameters and the internal length scale parameters used in Mindlin's Form II gradient elastic theory.

Nevertheless, in the aforementioned studies cortical bone was modeled as a two dimensional elastic plate. However Protopappas et al. [39] by using the classical theory of elasticity have shown that the cylindrical geometry of bone significantly affects the propagation of the higher order guided modes. To this end, the present study could be regarded as a step towards realistic modeling of the wave propagation in cortical bone exhibiting microstructure 
since for the first time the gradient theory of elasticity incorporating the full set of microstructural length scale parameters is used to model analytically guided wave propagation in three dimensional cylindrical geometry.

\section{Gradient elasticity in cylindrical geometry}

Mindlin in [2] suggested several forms concerning the variation of energy with strains and microstructural variables in materials with gradient features. The most appropriate form in elastic propagation in the framework of the long-wave length approximation, turns out to be form II of Mindlin [2] according to which the variation of the energy (potential energy per unit-macrovolume) becomes (see [1]) (using Einstein's summation convention)

$$
\delta U=\tau_{i j} \delta \epsilon_{i j}+\mu_{i j k} \delta \kappa_{i j k}
$$

where $\epsilon_{i j}$ is the usual strain (now the macro-strain), $\tau_{i j}$ the "macroscopic" Cauchy stress, $\mu_{i j k}$ the double stress tensor and $\kappa_{i j k} \equiv \partial_{i} \epsilon_{j k}$ is the gradient of the macro-stress tensor.

It is preferable to work with dyadic displacements [28] since it helps in incorporating uniformly all possible polarizations and in building a framework suitable to study Green's functions as well. We introduce thus the dyadic displacement field $\widetilde{u}=\mathbf{u}_{l} \widehat{\mathbf{x}}_{l}$ where $\mathbf{u}_{l}=u_{j l} \widehat{\mathbf{x}}_{j}$ are the cartesian vector components. The macro-strain tensor $\widetilde{e}=\frac{1}{2}\left(\nabla \widetilde{u}+(\nabla \widetilde{u})^{213}\right)$ is now a triadic [1] and so is the Cauchy stress tensor $\widetilde{\tau}$. The superscript (213) stands for a specific permutation of the components of the triadic to which it refers. For example $\left(\widehat{\mathbf{x}}_{\mathbf{i}} \widehat{\mathbf{x}}_{\mathbf{j}} \widehat{\mathbf{x}}_{\mathbf{k}}\right)^{213}=\widehat{\mathbf{x}}_{\mathbf{j}} \widehat{\mathbf{x}}_{\mathbf{i}} \widehat{\mathbf{x}}_{\mathbf{k}}$. Relevant symbolism is valid for higher order dyads. The gradient of the strain $(\nabla \widetilde{e})$ becomes now a tensor of fourth order and this property is inherited to the double stress tetradic: $\widetilde{\boldsymbol{\mu}}=\mu_{i j k l} \widehat{\mathbf{x}}_{i} \widehat{\mathbf{x}}_{j} \widehat{\mathbf{x}}_{k} \widehat{\mathbf{x}}_{l}$. The constitutive relations connecting strains and stresses are given in [2] and reflect the simplest possible assumption of a homogeneous, isotropic, quadratic (in terms of its arguments) energy density. More precisely, we express the Cauchy and double stresses as follows

$$
\widetilde{\boldsymbol{\tau}}=2 \mu \widetilde{e}+\lambda \widetilde{\mathbf{I}}(\nabla \cdot \widetilde{u})=\mu\left(\nabla \widetilde{u}+(\nabla \widetilde{u})^{213}\right)+\lambda \widetilde{I}(\nabla \cdot \widetilde{u}),
$$

and

$$
\begin{aligned}
\widetilde{\boldsymbol{\mu}} & =\frac{1}{2} a_{1}\left[(\widetilde{\mathbf{I}} \Delta \widetilde{u})^{3124}+\widetilde{\mathbf{I}} \nabla \nabla \cdot \widetilde{u}+(\widetilde{\mathbf{I}} \nabla \nabla \cdot \widetilde{u})^{3124}+(\widetilde{\mathbf{I}} \nabla \nabla \cdot \widetilde{u})^{1324}\right] \\
& +2 a_{2}(\widetilde{\mathbf{I}} \nabla \nabla \cdot \widetilde{u})^{3124}+\frac{1}{2} a_{3}\left[\widetilde{\mathbf{I}} \Delta \widetilde{u}+\widetilde{\mathbf{I}} \nabla \nabla \cdot \widetilde{u}+(\widetilde{\mathbf{I}} \Delta \widetilde{u})^{1324}+(\widetilde{\mathbf{I}} \nabla \nabla \cdot \widetilde{u})^{1324}\right] \\
& +a_{4}\left[\nabla \nabla \widetilde{u}+(\nabla \nabla \widetilde{u})^{2314}\right]+\frac{1}{2} a_{5}\left[2(\nabla \nabla \widetilde{u})^{3124}+\nabla \nabla \widetilde{u}+(\nabla \nabla \widetilde{u})^{2314}\right]
\end{aligned}
$$

where $\mu=\mu_{m a c}-\frac{2 g_{2}^{2}}{b_{2}+b_{3}}, \lambda+2 \mu=\lambda_{m a c}+2 \mu_{m a c}-\frac{8 g_{2}^{2}}{3\left(b_{2}+b_{3}\right)}-\frac{\left(3 g_{1}+2 g_{2}\right)^{2}}{3\left(3 b_{1}+b_{2}+b_{3}\right)}$. The parameters $\lambda_{\text {mac }}, \mu_{\text {mac }}$ are the Lame constants of the macroscopic elastic material, while $a_{i}, i=1, . .5, g_{i}, i=1,2$ and $b_{i}, i=1,2,3$ are constitutive parameters 
due exclusively to the presence of the microstructure. These internal length scale parameters are correlated to the bone microstructure. Their determination is one of the most demanding issues of relevant experiments. However we mention here corresponding works [38],[42] in which these coefficients or rather the produced parameters $h_{1}, h_{2}, g_{1}, g_{2}$ appeared later in the governing law (15), are expressed explicitly in terms of the structure of the cortical bone.

The variation of the potential energy $U_{V}$ stored in region every region $V$ of the material is given by the formula

$$
\delta U_{V}=\int_{V} \delta U d \mathbf{r}=\int_{V}\left[\widetilde{\boldsymbol{\tau}}: \delta \widetilde{\mathbf{e}}+(\widetilde{\boldsymbol{\mu}})^{4321}:: \delta \nabla \widetilde{\mathbf{e}}\right] d \mathbf{r}
$$

where the dots represent tensor contractions.

The differential equations along with the accompanying boundary conditions on the surface $S$ confining region $V$ for the dynamic gradient elasticity problem are produced via the application of Hamilton's principle

$$
\delta \int_{t_{0}}^{t_{1}}\left(K_{V}-U_{V}\right) d t+\int_{t_{0}}^{t_{1}} \delta W_{V} d t=0 .
$$

In the previous relation $K_{V}$ is the total kinetic energy of the medium occupying the volume $V$, while $\delta W_{V}$ is the variation of the work done by external forces expressed as [2], [4]

$$
\delta W_{V}=\int_{V} \widetilde{f}: \delta \widetilde{u}^{T} d \mathbf{r}+\int_{S} \widetilde{R}: \widehat{\mathbf{n}} \cdot \nabla\left(\delta \widetilde{u}^{T}\right) d S+\int_{S} \widetilde{P}: \delta \widetilde{u}^{T} d S
$$

where $\widetilde{f}$ denotes body forces, $\widetilde{P}$ surface tractions and $\widetilde{R}$ stands for surface double stresses.

Starting with Eq.(4), using Eqs.(5,6), adopting a suitable form for the kinetic energy and exploiting the calculus of variations, we can deduce the governing differential equations along with the accompanying boundary conditions. There are several models for the kinetic energy depending on the nature of the microstructure. Following here the approach of Mindlin, where there exists a not negligible contribution to the macro-velocity tensor from the micro-velocity field, which is actually proportional to the macro-velocity gradients, we infer that the differential equation of the gradient elasticity problem is

$$
\nabla \cdot(\widetilde{\boldsymbol{\tau}}-\nabla \cdot \widetilde{\boldsymbol{\mu}})+\widetilde{f}=\rho \frac{\partial^{2} \widetilde{u}}{\partial t^{2}}-\nabla \cdot\left(\rho^{\prime} \widetilde{D}: \frac{\partial^{2}}{\partial t^{2}} \nabla \widetilde{u}\right), \mathbf{r} \in V
$$

where $\rho^{\prime}$ stands for the mass of micro-material per unit macro-volume, $\rho$ is the macroscopic mass density and $\widetilde{D}$ is a specific tensor of fourth order depending on the physical and geometrical parameters of the microstructure. Its specific form can be found in references [1] (Eqs. (20,21)) or [2] (Eq. (9.27)) .

The corresponding boundary conditions are the following (where we recognize the surface gradient $\nabla_{S}$ ) 
i) classical BC's

$$
\begin{aligned}
& \widetilde{P}(\mathbf{r})=\widehat{\mathbf{n}} \cdot \widetilde{\boldsymbol{\tau}}(\mathbf{r})-\widehat{\mathbf{n}} \otimes \widehat{\mathbf{n}}: \frac{\partial \widetilde{\boldsymbol{\mu}}}{\partial n}(\mathbf{r})-\widehat{\mathbf{n}} \cdot\left(\nabla_{S} \cdot \widetilde{\boldsymbol{\mu}}(\mathbf{r})\right)-\widehat{\mathbf{n}} \cdot\left(\nabla_{S} \cdot \widetilde{\boldsymbol{\mu}}^{2134}(\mathbf{r})\right) \\
& +\left[\left(\nabla_{S} \cdot \widehat{\mathbf{n}}\right) \widehat{\mathbf{n}} \otimes \widehat{\mathbf{n}}-\left(\nabla_{S} \widehat{\mathbf{n}}\right)\right]: \widetilde{\boldsymbol{\mu}}(\mathbf{r}) \\
& +\widehat{\mathbf{n}} \cdot \rho^{\prime} \widetilde{D}: \frac{\partial^{2}}{\partial t^{2}}\left(\widehat{\mathbf{n}} \frac{\partial}{\partial n} \widetilde{u}(\mathbf{r})+\nabla_{S} \widetilde{u}(\mathbf{r})\right)=\widetilde{P}_{0}, \mathbf{r} \in S
\end{aligned}
$$

and/or

$$
\widetilde{u}(\mathbf{r})=\widetilde{u}_{0}, \mathbf{r} \in S
$$

ii) non classical BC's

$$
\widetilde{R}(\mathbf{r})=\widehat{\mathbf{n}} \cdot \widetilde{\boldsymbol{\mu}}^{1243}(\mathbf{r}) \cdot \widehat{\mathbf{n}}=\widetilde{R}_{0}, \mathbf{r} \in S
$$

and/or

$$
\frac{\partial \widetilde{u}}{\partial n}(\mathbf{r})=\widetilde{q}_{0}, \mathbf{r} \in S
$$

The fields $\widetilde{P}_{0}, \widetilde{u}_{0}, \widetilde{R}_{0}$ and $\widetilde{q}_{0}$ denote prescribed values.

The contribution $\widetilde{s}=-\nabla \cdot \widetilde{\boldsymbol{\mu}}$ to the classical stress tensor in Eq.(7), is given by

$$
\widetilde{s}=-\nabla \cdot \widetilde{\mu}=-\left[2 \mu c_{3} \Delta \widetilde{e}+\lambda c_{1} \widetilde{\mathbf{I}} \Delta(\nabla \cdot \widetilde{u})+\lambda c_{2} \nabla \nabla(\nabla \cdot \widetilde{u})\right]
$$

where $2 \mu c_{3}=a_{3}+2 a_{4}+a_{5}, \lambda c_{1}=a_{1}+2 a_{2}, \lambda c_{2}=a_{1}+a_{3}+a_{5}$.

We express the inertia term $\nabla \cdot\left(\rho^{\prime} \widetilde{D}: \frac{\partial^{2}}{\partial t^{2}} \nabla \widetilde{u}\right)$ as follows [1]

$$
\nabla \cdot\left(\rho^{\prime} \widetilde{D}: \frac{\partial^{2}}{\partial t^{2}} \nabla \widetilde{u}\right)=\rho \frac{\partial^{2}}{\partial t^{2}}\left(h_{1}^{2} \nabla \nabla \cdot \widetilde{u}-h_{2}^{2} \nabla \times \nabla \times \widetilde{u}\right)
$$

while the surface contribution $\widehat{\mathbf{n}} \cdot \rho^{\prime} \widetilde{D}: \frac{\partial^{2}}{\partial t^{2}}\left(\widehat{\mathbf{n}} \frac{\partial}{\partial n} \widetilde{u}+\nabla_{S} \widetilde{u}\right)$ in surface traction $\widetilde{P}$ becomes after some (vector) operators manipulations (see again [1])

$$
\begin{array}{r}
\widehat{\mathbf{n}} \cdot \rho^{\prime} \widetilde{D}: \frac{\partial^{2}}{\partial t^{2}}\left(\widehat{\mathbf{n}} \frac{\partial}{\partial n} \widetilde{u}+\nabla_{S} \widetilde{u}\right)=\rho \frac{\partial^{2}}{\partial t^{2}}\left[\left(h_{2}^{2}-\frac{\rho^{\prime} d^{2}}{3 \rho}\right) \widehat{\mathbf{n}} \times(\nabla \times \widetilde{u})\right. \\
\left.+\quad\left(2 h_{2}^{2}-\frac{\rho^{\prime} d^{2}}{3 \rho}\right) \frac{\partial \widetilde{u}}{\partial n}+\left(h_{1}^{2}-2 h_{2}^{2}+\frac{\rho^{\prime} d^{2}}{3 \rho}\right) \widehat{\mathbf{n}}(\nabla \cdot \widetilde{u})\right]
\end{array}
$$

where $h_{1}^{2}, h_{2}^{2}$ are positive numbers depending on constitutive parameters of the micro-deformation field along with geometrical parameters of the representative cell of the microstructure. On the basis of Eqs. $(12,13)$, the differential equation (7) becomes

$$
\begin{aligned}
& (\lambda+2 \mu)\left(1-\xi_{1}^{2} \Delta\right) \nabla \nabla \cdot \widetilde{u}-\mu\left(1-\xi_{2}^{2} \Delta\right) \nabla \times \nabla \times \widetilde{u}= \\
& \rho \frac{\partial^{2}}{\partial t^{2}}\left[\widetilde{u}-h_{1}^{2} \nabla \nabla \cdot \widetilde{u}+h_{2}^{2} \nabla \times \nabla \times \widetilde{u}\right],
\end{aligned}
$$


where the involved parameters $\xi_{1}$ and $\xi_{2}$ are given by

$$
\xi_{1}^{2}=\frac{\lambda\left(c_{1}+c_{2}\right)+2 \mu c_{3}}{\lambda+2 \mu}, \xi_{2}^{2}=c_{3} .
$$

We consider the case of time harmonic waves with frequency $\omega$ traveling with time dependence: $\exp (-i \omega t)$. Every elastic field in the sequel denotes the time-reduced part of the corresponding total field.

To solve Eq.(15), we start with the general representation for an arbitrary dyadic field $\widetilde{u}([36])$

$$
\widetilde{u}=\nabla \nabla \phi+\nabla \nabla \times \mathbf{A}+\nabla \times \nabla \times \widetilde{G} .
$$

with a general scalar field $\phi$, a free divergence vector field $\mathbf{A}$ and a dyadic field $\widetilde{G}$. We substitute the expansion (17) in Eq.(15) and separate the corresponding terms based on properties of solenoidal and irrotational fields. Following the same theoretical process as in [1], we find that the first part of this decomposition is contained in the large set

$$
\nabla \nabla \phi \in\left\{\nabla \mathbf{L}^{m, l}\left(\mathbf{r} ; k_{i}, \xi\right) i=1,2, m=0, \pm 1, \pm 2, \ldots, l=1,2,3,4\right\},
$$

where

$$
\begin{aligned}
& k_{1}^{2}(\omega)=\frac{2 \rho \omega^{2}}{(\lambda+2 \mu)-\rho h_{1}^{2} \omega^{2}+\sqrt{D(\omega)}} \\
& k_{2}^{2}(\omega)=\frac{2 \rho \omega^{2}}{(\lambda+2 \mu)-\rho h_{1}^{2} \omega^{2}-\sqrt{D(\omega)}}
\end{aligned}
$$

while the second part is characterized as

$$
\begin{aligned}
& \nabla \nabla \times \mathbf{A} \in\left\{\nabla \mathbf{M}^{m, l}\left(\mathbf{r} ; k_{i}, \xi\right) ; i=1,2, m=0, \pm 1, \pm 2, \ldots, l=1,2,3,4\right\} \\
& \cup\left\{\nabla \mathbf{N}^{m, l}\left(\mathbf{r} ; k_{i}, \xi\right) ; i=1,2, m=0, \pm 1, \pm 2, \ldots, l=1,2,3,4\right\} .
\end{aligned}
$$

Note [1] that $D(\omega)=\left(\lambda+2 \mu-\rho h_{1}^{2} \omega^{2}\right)^{2}+4 \rho \omega^{2} \xi_{1}^{2}(\lambda+2 \mu)$.

To introduce some necessary terminology, we define here the Navier eigenvectors $\mathbf{L}, \mathbf{M}, \mathbf{N}$, which expressed in cylindrical coordinates $r, \phi, z$, have the form (consult [29] for some diversification w.r.t the spherical geometry)

$$
\begin{aligned}
\mathbf{L}^{m, l}(\mathbf{r} ; k, \xi) & =\frac{1}{\xi} \nabla \chi^{m, l}(\mathbf{r} ; k, \xi) \\
\mathbf{M}^{m, l}(\mathbf{r} ; k, \xi) & =\frac{1}{\xi} \nabla \times\left(\hat{\mathbf{z}} \chi^{m, l}(\mathbf{r} ; k, \xi)\right) \\
\mathbf{N}^{m, l}(\mathbf{r} ; k, \xi) & =\frac{1}{\xi^{2}} \frac{\partial}{\partial z} \nabla \times \mathbf{M}_{n}^{m, l}(\mathbf{r} ; k)
\end{aligned}
$$

where $\chi^{m, l}(\mathbf{r} ; k, \xi)=S_{m}^{l}(\xi r) \Phi^{m}(\phi) Z(z ; p)$ is the general separable solution of Helmholtz equation corresponding to wave number $k$ with $k^{2}-p^{2}=\xi^{2}$. As a matter of fact $S_{m}^{l}$ stands for the cylindrical Bessel (Newmann) functions of 
order $m$ for $l=1(l=2)$, or alternatively for the Hankel functions $(l=3,4)$. Furthermore $\Phi^{m}(\phi)=\exp (i m \phi)$ and $Z(z ; p)=\exp (i p z)$. Several cases emerge concerning the behavior of these eigensolutions in $r$ and $z$ directions. The crucial relation is $k^{2}=\xi^{2}+p^{2}$, which divides the (square of the) wave number $k^{2}$ into the polar plus the axial portion and creates many possibilities for propagating or attenuated waves. Remark that the elements of the sets (18),(21) with $i=2$ correspond to negative $k^{2}$, i.e. to imaginary wave number $k$, which is reasonable in the framework of gradient elasticity.

The third term of the decomposition (17) can be constructed exactly as in [1] and is characterized as follows:

$$
\begin{aligned}
& \nabla \times(\nabla \times \widetilde{G}) \in \frac{1}{\xi}\left\{\nabla \times\left(\mathbf{L}^{m, l}\left(\mathbf{r}, k_{j}, \xi\right) \times \widetilde{\mathbf{I}}\right), \nabla \times\left(\mathbf{M}^{m, l}\left(\mathbf{r}, k_{j}, \xi\right) \times \widetilde{\mathbf{I}}\right),\right. \\
& \nabla \times\left(\mathbf{N}^{m, l}\left(\mathbf{r}, k_{j}, \xi\right) \times \widetilde{\mathbf{I}}\right), \nabla \times\left(\hat{\mathbf{z}} \mathbf{L}^{m, l}\left(\mathbf{r}, k_{j}, \xi\right)\right), \\
& \nabla \times\left(\hat{\mathbf{z}} \mathbf{M}^{m, l}\left(\mathbf{r}, k_{j}, \xi\right)\right), \nabla \times\left(\hat{\mathbf{z}} \mathbf{N}^{m, l}\left(\mathbf{r}, k_{j}, \xi\right)\right), \quad j=3,4 \\
& m=0, \pm 1, \pm 2, . ., ; l=1,2,3,4\}
\end{aligned}
$$

where

$$
k_{3}^{2}(\omega)=\frac{2 \rho \omega^{2}}{\mu-\rho h_{2}^{2} \omega^{2}+\sqrt{D^{\prime}(\omega)}}, k_{4}^{2}(\omega)=\frac{2 \rho \omega^{2}}{\mu-\rho h_{2}^{2} \omega^{2}-\sqrt{D^{\prime}(\omega)}}
$$

with $D^{\prime}(\omega)=\left(\mu-\rho h_{2}^{2} \omega^{2}\right)^{2}+4 \rho \omega^{2} \xi_{2}^{2} \mu$. Remark again that $k_{4}$ is imaginary. The multiplicative factor $1 / \xi$ whenever it appears functions as a scaling parameter, conserving the non-dimensional structure (w.r.t the polar direction size) of the basis functions.

Via the Eqs.(17,18,21) and Eq.(25), we have determined the fundamental eigendyadics constituting the structural solutions of the dynamic gradient elasticity. For every specific azimouthal integer $m$, every generic complex $z$ wavenumber $p$ and every concrete frequency $\omega$, we deal with nine dyadic eigensolutions which constitute the analog of the Navier eigenvectors in the dyadic framework. They can thus be called Navier eigendyadics indicating their relevance to the vector case. Every one of these nine eigendyadics corresponds to two possible wavenumbers (one real and one imaginary). The first two parts of Eq.(17) contains irrotational eigendyadics referring to two "longitudinal-type" wave numbers while the third part involves solenoidal eigendyadics characterized by "shear-type" wavenumbers.

It is very important to express these eigensolutions in separable forms of cylindrical coordinates. This is necessary first in order to handle boundary value problems described geometrically even approximately by the cylindrical coordinate system. In addition, in the framework of the integral equation setting, it is essential to express the integral kernels in terms of these separable dyadic solutions. Following reference [1], after extended and very involved manipulations, we have constructed the forthcoming separable cylindrical representations for the aforementioned dyadic solutions. Special role in these representations play 
the cylindrical vector harmonics [29]

$$
\begin{aligned}
& \mathbf{P}_{m}(\phi, z ; p)=\hat{\mathbf{r}} e^{i m \phi} Z(z ; p) \\
& \mathbf{B}_{m}(\phi, z ; p)=\hat{\phi} e^{i m \phi} Z(z ; p) \\
& \mathbf{C}_{m}(\phi, z ; p)=i \hat{\mathbf{z}} e^{i m \phi} Z(z ; p)
\end{aligned}
$$

The aforementioned representations obey the following relations:

$$
\begin{aligned}
& \frac{\nabla \mathbf{L}}{\xi}=\ddot{S}_{m}^{l}(\xi r)\left(\hat{\mathbf{r}} \mathbf{P}_{m}\right)+2 i m \frac{d}{d(\xi r)}\left(\frac{S_{m}^{l}(\xi r)}{\xi r}\right)\left(\hat{\mathbf{r}} \mathbf{B}_{m}\right)_{s} \\
& +\left(\frac{\dot{S}_{m}^{l}(\xi r)}{\xi r}-m^{2} \frac{S_{m}^{l}(\xi r)}{(\xi r)^{2}}\right)\left(\hat{\phi} \mathbf{B}_{m}\right)+2 \frac{p}{\xi} \dot{S}_{m}^{l}(\xi r)\left(\hat{\mathbf{r}} \mathbf{C}_{m}\right)_{s} \\
& +2 i m \frac{p}{\xi} \frac{S_{m}^{l}(\xi r)}{\xi r}\left(\hat{\phi} \mathbf{C}_{m}\right)_{s}-\frac{p^{2}}{\xi^{2}} S_{m}^{l}(\xi r)\left(-i \hat{\mathbf{z}} \mathbf{C}_{m}\right)
\end{aligned}
$$

$$
\begin{aligned}
& \frac{\nabla \mathbf{M}}{\xi}= \\
& i m \frac{d}{d(\xi r)}\left(\frac{S_{m}^{l}(\xi r)}{\xi r}\right)\left(\hat{\mathbf{r}} \mathbf{P}_{m}\right)-i m \frac{d}{d(\xi r)}\left(\frac{S_{m}^{l}(\xi r)}{\xi r}\right)\left(\hat{\phi} \mathbf{B}_{m}\right)-\frac{p}{\xi} \dot{S}_{m}^{l}(\xi r)\left(\hat{\phi} \mathbf{C}_{m}\right)_{s} \\
& {\left[2\left(\frac{\dot{S}_{m}^{l}(\xi r)}{\xi r}-\frac{m^{2}}{(\xi r)^{2}} S_{m}^{l}(\xi r)\right)+S_{m}^{l}(\xi r)\right]\left(\hat{\mathbf{r}} \mathbf{B}_{m}\right)_{s}+i m \frac{p}{\xi} \frac{S_{m}^{l}(\xi r)}{\xi r}\left(\hat{\mathbf{r}} \mathbf{C}_{m}\right)_{s}} \\
& +S_{m}^{l}(\xi r)\left(\hat{\mathbf{r}} \mathbf{B}_{m}\right)_{\alpha}-i m \frac{p}{\xi} \frac{S_{m}^{l}(\xi r)}{\xi r}\left(\hat{\mathbf{r}} \mathbf{C}_{m}\right)_{\alpha}+\frac{p}{\xi} \dot{S}_{m}^{l}(\xi r)\left(\hat{\phi} \mathbf{C}_{m}\right)_{\alpha} \\
& \quad \frac{\nabla \mathbf{N}}{\xi}=-\frac{p^{2}}{\xi^{2}} \ddot{S}_{m}^{l}(\xi r)\left(\hat{\mathbf{r}} \mathbf{P}_{m}\right)-2 i m \frac{p^{2}}{\xi^{2}} \frac{d}{d(\xi r)}\left(\frac{S_{m}^{l}(\xi r)}{\xi r}\right)\left(\hat{\mathbf{r}} \mathbf{B}_{m}\right)_{s} \\
& \quad-\frac{p^{2}}{\xi^{2}}\left(\frac{\dot{S}_{m}^{l}(\xi r)}{\xi r}-\frac{m^{2}}{(\xi r)^{2}} S_{m}^{l}(\xi r)\right)\left(\hat{\phi} \mathbf{B}_{m}\right)+\frac{p}{\xi}\left(1-\frac{p^{2}}{\xi^{2}}\right) \dot{S}_{m}^{l}(\xi r)\left(\hat{\mathbf{r}} \mathbf{C}_{m}\right)_{s} \\
& \quad+i m \frac{p}{\xi}\left(1-\frac{p^{2}}{\xi^{2}}\right) \frac{S_{m}^{l}(\xi r)}{\xi r}\left(\hat{\phi} \mathbf{C}_{m}\right)_{s}-\frac{p^{2}}{\xi^{2}} S_{m}^{l}(\xi r)\left(-i \hat{\mathbf{z}} \mathbf{C}_{m}\right) \\
& \quad+\frac{p}{\xi} \frac{k^{2}}{\xi^{2}} \dot{S}_{m}^{l}(\xi r)\left(\hat{\mathbf{r}} \mathbf{C}_{m}\right)_{\alpha}+i m \frac{p}{\xi} \frac{k^{2}}{\xi^{2}} \frac{S_{m}^{l}(\xi r)}{\xi r}\left(\hat{\phi} \mathbf{C}_{m}\right)_{\alpha}
\end{aligned}
$$




$$
\begin{aligned}
& \frac{\nabla \times(\mathbf{L} \times \tilde{\mathbf{I}})}{\xi}= \\
& =\frac{1}{\xi^{2}} \nabla \nabla \chi^{m, l}+\frac{k^{2}}{\xi^{2}} \chi^{m, l} \tilde{\mathbf{I}}=\frac{\nabla \mathbf{L}}{\xi}+\frac{k^{2}}{\xi^{2}}\left(\chi^{m, l} \hat{\mathbf{r}} \hat{\mathbf{r}}+\chi^{m, l} \hat{\phi} \hat{\phi}+\chi^{m, l} \hat{\mathbf{z}} \hat{\mathbf{z}}\right) \\
& =\left(\ddot{S}_{m}^{l}(\xi r)+\frac{k^{2}}{\xi^{2}} S_{m}^{l}(\xi r)\right)\left(\hat{\mathbf{r}} \mathbf{P}_{m}\right)+2 i m \frac{d}{d(\xi r)}\left(\frac{S_{m}^{l}(\xi r)}{\xi r}\right)\left(\hat{\mathbf{r}} \mathbf{B}_{m}\right)_{s} \\
& +\left(\frac{\dot{S}_{m}^{l}(\xi r)}{\xi r}+\left(\frac{k^{2}}{\xi^{2}}-\frac{m^{2}}{(\xi r)^{2}}\right) S_{m}^{l}(\xi r)\right)\left(\hat{\phi} \mathbf{B}_{m}\right)+2 \frac{p}{\xi} \dot{S}_{m}^{l}(\xi r)\left(\hat{\mathbf{r}} \mathbf{C}_{m}\right)_{s} \\
& +2 i m \frac{p}{\xi} \frac{S_{m}^{l}(\xi r)}{\xi r}\left(\hat{\phi} \mathbf{C}_{m}\right)_{s}+S_{m}^{l}(\xi r)\left(-i \hat{\mathbf{z}} \mathbf{C}_{m}\right) \\
& \frac{\nabla \times(\mathbf{M} \times \tilde{\mathbf{I}})}{\xi}=\frac{\left(\nabla \mathbf{M}^{T}\right)}{\xi} \\
& =i m \frac{d}{d(\xi r)}\left(\frac{S_{m}^{l}(\xi r)}{\xi r}\right)\left(\hat{\mathbf{r}} \mathbf{P}_{m}\right)-i m \frac{d}{d(\xi r)}\left(\frac{S_{m}^{l}(\xi r)}{\xi r}\right)\left(\hat{\phi} \mathbf{B}_{m}\right)-\frac{p}{\xi} \dot{S}_{m}^{l}(\xi r)\left(\hat{\phi} \mathbf{C}_{m}\right)_{s} \\
& {\left[2\left(\frac{\dot{S}_{m}^{l}(\xi r)}{\xi r}-\frac{m^{2}}{(\xi r)^{2}} S_{m}^{l}(\xi r)\right)+S_{m}^{l}(\xi r)\right]\left(\hat{\mathbf{r}} \mathbf{B}_{m}\right)_{s}+i m \frac{p}{\xi} \frac{S_{m}^{l}(\xi r)}{\xi r}\left(\hat{\mathbf{r}} \mathbf{C}_{m}\right)_{s}} \\
& -S_{m}^{l}(\xi r)\left(\hat{\mathbf{r}} \mathbf{B}_{m}\right)_{\alpha}+i m \frac{p}{\xi} \frac{S_{m}^{l}(\xi r)}{\xi r}\left(\hat{\mathbf{r}} \mathbf{C}_{m}\right)_{\alpha}-\frac{p}{\xi} \dot{S}_{m}^{l}(\xi r)\left(\hat{\phi} \mathbf{C}_{m}\right)_{\alpha} \\
& \frac{\nabla \times(\mathbf{N} \times \tilde{\mathbf{I}})}{\xi}=\frac{\left(\nabla \mathbf{N}^{T}\right)}{\xi} \\
& =-\frac{p^{2}}{\xi^{2}} \ddot{S}_{m}^{l}(\xi r)\left(\hat{\mathbf{r}} \mathbf{P}_{m}\right)-2 i m \frac{p^{2}}{\xi^{2}} \frac{d}{d(\xi r)}\left(\frac{S_{m}^{l}(\xi r)}{\xi r}\right)\left(\hat{\mathbf{r}} \mathbf{B}_{m}\right)_{s} \\
& -\frac{p^{2}}{\xi^{2}}\left(\frac{\dot{S}_{m}^{l}(\xi r)}{\xi r}-\frac{m^{2}}{(\xi r)^{2}} S_{m}^{l}(\xi r)\right)\left(\hat{\phi} \mathbf{B}_{m}\right)+\frac{p}{\xi}\left(1-\frac{p^{2}}{\xi^{2}}\right) \dot{S}_{m}^{l}(\xi r)\left(\hat{\mathbf{r}} \mathbf{C}_{m}\right)_{s} \\
& +i m \frac{p}{\xi}\left(1-\frac{p^{2}}{\xi^{2}}\right) \frac{S_{m}^{l}(\xi r)}{\xi r}\left(\hat{\phi} \mathbf{C}_{m}\right)_{s}-\frac{p^{2}}{\xi^{2}} S_{m}^{l}(\xi r)\left(-i \hat{\mathbf{z}} \mathbf{C}_{m}\right) \\
& -\frac{p}{\xi} \frac{k^{2}}{\xi^{2}} \dot{S}_{m}^{l}(\xi r)\left(\hat{\mathbf{r}} \mathbf{C}_{m}\right)_{\alpha}-i m \frac{p}{\xi} \frac{k^{2}}{\xi^{2}} \frac{S_{m}^{l}(\xi r)}{\xi r}\left(\hat{\phi} \mathbf{C}_{m}\right)_{\alpha}
\end{aligned}
$$




$$
\begin{aligned}
& \frac{1}{\xi} \nabla \times(\hat{\mathbf{z}} \mathbf{L})=-\frac{1}{\xi} \hat{\mathbf{z}} \times \nabla \mathbf{L} \\
& \left(=-\ddot{S}_{m}^{l}(\xi r)\left(\left(\hat{\mathbf{r}} \mathbf{B}_{m}\right)_{s}-\left(\hat{\mathbf{r}} \mathbf{B}_{m}\right)_{\alpha}\right)-i m \frac{d}{d(\xi r)}\left(\frac{S_{m}^{l}(\xi r)}{\xi r}\right)\left(\hat{\phi} \mathbf{B}_{m}-\hat{\mathbf{r}} \mathbf{P}_{m}\right)\right. \\
& \left(\frac{\dot{S}_{m}^{l}(\xi r)}{\xi r}-m^{2} \frac{S_{m}^{l}(\xi r)}{(\xi r)^{2}}\right)\left(\left(\hat{\mathbf{r}} \mathbf{B}_{m}\right)_{s}+\left(\hat{\mathbf{r}} \mathbf{B}_{m}\right)_{\alpha}\right)-\frac{p}{\xi} \dot{S}_{m}^{l}(\xi r)\left(\left(\hat{\phi} \mathbf{C}_{m}\right)_{s}+\left(\hat{\phi} \mathbf{C}_{m}\right)_{\alpha}\right) \\
& \left.+i m \frac{p}{\xi} \frac{S_{m}^{l}(\xi r)}{\xi r}\left(\left(\hat{\mathbf{r}} \mathbf{C}_{m}\right)_{s}+\left(\hat{\mathbf{r}} \mathbf{C}_{m}\right)_{\alpha}\right)\right) \\
& =\left[S_{m}^{l}(\xi r)+2\left(\frac{\dot{S}_{m}^{l}(\xi r)}{\xi r}-m^{2} \frac{S_{m}^{l}(\xi r)}{(\xi r)^{2}}\right)\right]\left(\hat{\mathbf{r}} \mathbf{B}_{m}\right)_{s}-S_{m}^{l}(\xi r)\left(\hat{\mathbf{r}} \mathbf{B}_{m}\right)_{\alpha} \\
& -i m \frac{d}{d(\xi r)}\left(\frac{S_{m}^{l}(\xi r)}{\xi r}\right)\left(\hat{\phi} \mathbf{B}_{m}-\hat{\mathbf{r}} \mathbf{P}_{m}\right)-\frac{p}{\xi} \dot{S}_{m}^{l}(\xi r)\left(\left(\hat{\phi} \mathbf{C}_{m}\right)_{s}+\left(\hat{\phi} \mathbf{C}_{m}\right)_{\alpha}\right) \\
& +i m \frac{p}{\xi} \frac{S_{m}^{l}(\xi r)}{\xi r}\left(\left(\hat{\mathbf{r}} \mathbf{C}_{m}\right)_{s}+\left(\hat{\mathbf{r}} \mathbf{C}_{m}\right)_{\alpha}\right) \\
& \frac{1}{\xi} \nabla \times(\hat{\mathbf{z}} \mathbf{M})=-\frac{1}{\xi} \hat{\mathbf{z}} \times \nabla \mathbf{M} \\
& \left(=-i m \frac{d}{d(\xi r)}\left(\frac{S_{m}^{l}(\xi r)}{\xi r}\right)\left(\mathbf{B}_{m} \hat{\mathbf{r}}\right)-i m \frac{d}{d(\xi r)}\left(\frac{S_{m}^{l}(\xi r)}{\xi r}\right)\left(\hat{\mathbf{r}} \mathbf{B}_{m}\right)\right. \\
& -\frac{1}{2}\left[2\left(\frac{\dot{S}_{m}^{l}(\xi r)}{\xi r}-\frac{m^{2}}{(\xi r)^{2}} S_{m}^{l}(\xi r)\right)+S_{m}^{l}(\xi r)\right]\left(\left(\hat{\phi} \mathbf{B}_{m}\right)-\left(\hat{\mathbf{r}} \mathbf{P}_{m}\right)\right) \\
& \left.-\frac{1}{2} S_{m}^{l}(\xi r)\left(\left(\hat{\phi} \mathbf{B}_{m}\right)+\left(\hat{\mathbf{r}} \mathbf{P}_{m}\right)\right)\right) \\
& =-2 i m \frac{d}{d(\xi r)}\left(\frac{S_{m}^{l}(\xi r)}{\xi r}\right)\left(\hat{\mathbf{r}} \mathbf{B}_{m}\right)_{s}+\left(\frac{\dot{S}_{m}^{l}(\xi r)}{\xi r}-\frac{m^{2}}{(\xi r)^{2}} S_{m}^{l}(\xi r)\right)\left(\hat{\mathbf{r}} \mathbf{P}_{m}\right) \\
& -\left(\frac{\dot{S}_{m}^{l}(\xi r)}{\xi r}+\left(1-\frac{m^{2}}{(\xi r)^{2}}\right) S_{m}^{l}(\xi r)\right)\left(\hat{\phi} \mathbf{B}_{m}\right)
\end{aligned}
$$




$$
\begin{aligned}
& \frac{1}{\xi} \nabla \times(\hat{\mathbf{z}} \mathbf{N})=-\frac{1}{\xi} \hat{\mathbf{z}} \times \nabla \mathbf{N} \\
& \left(=+\frac{p^{2}}{\xi^{2}} \ddot{S}_{m}^{l}(\xi r)\left(\mathbf{B}_{m} \hat{\mathbf{r}}\right)+i m \frac{p^{2}}{\xi^{2}} \frac{d}{d(\xi r)}\left(\frac{S_{m}^{l}(\xi r)}{\xi r}\right)\left(\left(\hat{\phi} \mathbf{B}_{m}\right)-\left(\hat{\mathbf{r}} \mathbf{P}_{m}\right)\right)\right. \\
& -\frac{p^{2}}{\xi^{2}}\left(\frac{\dot{S}_{m}^{l}(\xi r)}{\xi r}-\frac{m^{2}}{(\xi r)^{2}} S_{m}^{l}(\xi r)\right)\left(\hat{\mathbf{r}} \mathbf{B}_{m}\right)-\frac{p}{\xi} \dot{S}_{m}^{l}(\xi r)\left(\hat{\phi} \mathbf{C}_{m}\right) \\
& \left.+i m \frac{p}{\xi} \frac{S_{m}^{l}(\xi r)}{\xi r}\left(\hat{\mathbf{r}} \mathbf{C}_{m}\right)\right) \\
& =i m \frac{p^{2}}{\xi^{2}} \frac{d}{d(\xi r)}\left(\frac{S_{m}^{l}(\xi r)}{\xi r}\right)\left(\left(\hat{\phi} \mathbf{B}_{m}\right)-\left(\hat{\mathbf{r}} \mathbf{P}_{m}\right)\right)+\frac{p^{2}}{\xi^{2}} S_{m}^{l}(\xi r)\left(\hat{\mathbf{r}} \mathbf{B}_{m}\right)_{\alpha} \\
& -\frac{p^{2}}{\xi^{2}}\left(2\left(\frac{\dot{S}_{m}^{l}(\xi r)}{\xi r}-\frac{m^{2}}{(\xi r)^{2}} S_{m}^{l}(\xi r)\right)+S_{m}^{l}(\xi r)\right)\left(\hat{\mathbf{r}} \mathbf{B}_{m}\right)_{s}-\frac{p}{\xi} \dot{S}_{m}^{l}(\xi r)\left(\left(\hat{\phi} \mathbf{C}_{m}\right)_{s}\right. \\
& \left.+\left(\hat{\phi} \mathbf{C}_{m}\right)_{\alpha}\right)+i m \frac{p}{\xi} \frac{S_{m}^{l}(\xi r)}{\xi r}\left(\left(\hat{\mathbf{r}} \mathbf{C}_{m}\right)_{s}+\left(\hat{\mathbf{r}} \mathbf{C}_{m}\right)_{\alpha}\right)
\end{aligned}
$$

We remark that the dyadic eigensolutions of the dynamic equation of gradient elasticity - after being restricted on a specific cylinder lateral surface- are expanded in terms of a set of dyadic cylindrical harmonics, which constitutes the extension of the vector spherical harmonics. For every specific azimouthal parameter $m$ and every value of the "continuous" variable $p$ (the $z$-component of the wavenumber), this set contains six symmetric and three antisymmetric elements. The symmetric elements are the following:

$$
\begin{aligned}
& \hat{\mathbf{r}} \mathbf{P}_{m},\left(\hat{\mathbf{r}} \mathbf{B}_{m}\right)_{s}=\frac{\hat{\mathbf{r}} \mathbf{B}_{m}+\mathbf{B}_{m} \hat{\mathbf{r}}}{2}, \quad \hat{\phi} \mathbf{B}_{m}, \quad\left(\hat{\mathbf{r}} \mathbf{C}_{m}\right)_{s}=\frac{\hat{\mathbf{r}} \mathbf{C}_{m}+\mathbf{C}_{m} \hat{\mathbf{r}}}{2}, \\
& \left(\hat{\phi} \mathbf{C}_{m}\right)_{s}=\frac{\hat{\phi} \mathbf{C}_{m}+\mathbf{C}_{m} \hat{\phi}}{2}, \quad-i \hat{\mathbf{z}} \mathbf{C}_{m}
\end{aligned}
$$

while the antisymmetric terms are the three dyadics:

$$
\begin{aligned}
\left(\hat{\mathbf{r}} \mathbf{B}_{m}\right)_{\alpha} & =\frac{\hat{\mathbf{r}} \mathbf{B}_{m}-\mathbf{B}_{m} \hat{\mathbf{r}}}{2}, \quad\left(\hat{\mathbf{r}} \mathbf{C}_{m}\right)_{\alpha}=\frac{\hat{\mathbf{r}} \mathbf{C}_{m}-\mathbf{C}_{m} \hat{\mathbf{r}}}{2}, \\
\left(\hat{\phi} \mathbf{C}_{m}\right)_{\alpha} & =\frac{\hat{\phi} \mathbf{C}_{m}-\mathbf{C}_{m} \hat{\phi}}{2} .
\end{aligned}
$$

It can be proved - much more easily than in the treatment of the spherical case handled in [1] - that the cylindrical harmonics under discussion are orthogonal with respect to the $L^{2}$-norm on the aforementioned lateral cylindrical surface. More precisely the induced inner product involves double contraction of the dyadic elements and integration over the lateral surface. 


\section{Wave propagation inside a cylindrical wave guide with microstructure}

In this section we apply the results of the spectral analysis introduced in the previous section to the solution of a representative wave propagation problem. We consider a cylindrical wave guide of radius $a$, occupying the region $D$, surrounded by the lateral surface $S$, infinitely extended in $z$-direction and being filled with an elastic macroscopically isotropic material with microstructure, fully characterized by the parameters $\lambda$ and $\mu$, the densities $\rho, \rho^{\prime}$, the gradient parameters $\alpha_{l}, l=1,2, . .5, h_{1}, h_{2}$, and the characteristic micro-dimension $d$.

We examine the possibility of propagation of an elastic time-harmonic monochromatic- dyadic elastic field of the form $\widetilde{u}(\mathbf{r}, t)=\widetilde{u}(\mathbf{r}) e^{-i \omega t}=\widetilde{u}_{\perp}(r, \phi)$ $e^{i(p z-\omega t)}$ inside $D$, clearly in the long direction of the cylinder. Here $\widetilde{u}_{\perp}(r, \phi)$ is the time-reduced cross section part of the field. It is worthwhile to notice that the dyadic form of this field permits to represent uniformly all the possible polarizations of the traveling field. As deduced from the analysis described in the previous section, the second and last vector in the representation $\widetilde{u}=$ $\mathbf{u}_{l} \widehat{\mathbf{x}}_{l}$ of the dyadic elastic field does not participate in algebraic (or differential) manipulations pertaining to the involved boundary value problems. It is then sufficient to "dot" the constructed dyadic elastic field -at the end of the dayon all three cartesian directions thus produce all the possible vector elastic propagating fields corresponding to every specific propagation pair $(\omega, p)$. We note however that the principal aim is to connect the parameters $\omega$ and $p$, i.e. to establish the dispersion relation of the wave propagation in the waveguide.

The time reduced part $\widetilde{u}(\mathbf{r})$ of the propagating dyadic mode can be expressed in terms of the complete set of dyadic cylindrical eigensolutions, produced in the previous section as follows

$$
\begin{aligned}
& \widetilde{u}(\cdot ; \omega, p)=\sum_{j=1}^{2} \sum_{m=-\infty}^{m=\infty}\left[a_{j}^{m} \frac{\nabla \mathbf{L}_{j}^{m, 1}}{\xi_{j}}+b_{j}^{m} \frac{\nabla \mathbf{M}_{j}^{m, 1}}{\xi_{j}}+c_{j}^{m} \frac{\nabla \mathbf{N}_{j}^{m, 1}}{\xi_{j}}\right] \\
& +\sum_{j=3}^{4} \sum_{m=-\infty}^{m=\infty}\left[\alpha_{j}^{m} \frac{\nabla \times\left(\mathbf{L}_{j}^{m, 1} \times \tilde{\mathbf{I}}\right)}{\xi_{j}}+\beta_{j}^{m} \frac{\nabla \times\left(\mathbf{M}_{j}^{m, 1} \times \tilde{\mathbf{I}}\right)}{\xi_{j}}\right. \\
& +\gamma_{j}^{m} \frac{\nabla \times\left(\mathbf{N}_{j}^{m, 1} \times \tilde{\mathbf{I}}\right)}{\xi_{j}}+\delta_{j}^{m} \frac{\nabla \times\left(\hat{\mathbf{z}} \mathbf{L}_{j}^{m, 1}\right)}{\xi_{j}}+\epsilon_{j}^{m} \frac{\nabla \times\left(\hat{\mathbf{z}} \mathbf{M}_{j}^{m, 1}\right)}{\xi_{j}} \\
& \left.+\zeta_{j}^{m} \frac{\nabla \times\left(\hat{\mathbf{z}} \mathbf{N}_{j}^{m, 1}\right)}{\xi_{j}}\right] .
\end{aligned}
$$

The propagating field $\widetilde{u}$ given by (39) satisfies the boundary conditions $(8,10)$ in their homogeneous form, i.e.

$$
\begin{aligned}
\widetilde{P}(\mathbf{r}) & =0, \quad \mathbf{r} \in S \\
\widetilde{R}(\mathbf{r})\left(=\widehat{\mathbf{r}} \cdot \widetilde{\boldsymbol{\mu}}^{1243}(\mathbf{r}) \cdot \widehat{\mathbf{r}}\right) & =0, \quad \mathbf{r} \in S
\end{aligned}
$$


The surface traction $\widetilde{P}$ can be formed in the time-harmonic case by exploiting Eqs. $(8,14)$ on the lateral surface $S$ as follows

$$
\begin{aligned}
& \widetilde{P}(\mathbf{r})=\widehat{\mathbf{r}} \cdot \widetilde{\boldsymbol{\tau}}-\widehat{\mathbf{r}} \widehat{\mathbf{r}}: \frac{\partial \widetilde{\boldsymbol{\mu}}}{\partial r}-\widehat{\mathbf{r}} \cdot\left(\nabla_{S} \cdot \widetilde{\boldsymbol{\mu}}(\mathbf{r})\right)-\widehat{\mathbf{r}} \cdot\left(\nabla_{S} \cdot \widetilde{\boldsymbol{\mu}}^{2134}\right) \\
& +\left[\left(\nabla_{S} \cdot \widehat{\mathbf{r}}\right) \widehat{\mathbf{r}} \widehat{\mathbf{r}}-\nabla_{S} \widehat{\mathbf{r}}\right]: \widetilde{\boldsymbol{\mu}} \\
& -\rho \omega^{2}\left[\left(h_{2}^{2}-\frac{\rho^{\prime} d^{2}}{3 \rho}\right) \widehat{\mathbf{r}} \times(\nabla \times \widetilde{u})+\left(2 h_{2}^{2}-\frac{\rho^{\prime} d^{2}}{3 \rho}\right) \frac{\partial \widetilde{u}}{\partial r}\right. \\
& \left.+\left(h_{1}^{2}-2 h_{2}^{2}+\frac{\rho^{\prime} d^{2}}{3 \rho}\right) \widehat{\mathbf{r}}(\nabla \cdot \widetilde{u}(\mathbf{r}))\right]
\end{aligned}
$$

The treatment of the boundary conditions is realized in a classical systematic case given the set of the dyadic cylindrical harmonics. More precisely, we project functionally the conditions $(40,41)$ on these harmonics. This projection is equivalent to double contracting with those dyadic harmonics and integrating over the lateral surface. The propagating field $\widetilde{u}$ appearing in the boundary conditions is represented via Eq.(39) and admits of course several differential operations therein. Every generated separate term, confined to $r=a$, is an infinite expansion of the aforementioned dyadic harmonics. So the projection mechanism reveals the importance of mutual orthogonality of the dyadic harmonic functions. It is interesting that these orthogonality relations help to obtain separate algebraic systems for every index $m$. In other words the terms corresponding to different spectral parameters $m \in Z$ decouple. In addition only harmonic dyads pertaining to the wave parameter $p$ survive in the orthogonalization process.

It is clear from the form of the involved polyadics in the conditions $(40,41)$ that the analytic realization of the described above functional projection is demanding. However after tedious but straightforward analysis we are in position to obtain purely separate finite algebraic systems, each one of them involving exclusively the coefficients of the representation (39) referring to a specific spectral parameter $m$. Indeed Eqs. $(40,41)$ lead to the finite algebraic subsystems (everyone for specific $m=0, \pm 1, \pm 2, \ldots$ )

$$
\begin{aligned}
& \sum_{j=1}^{2}\left[A_{s, q, j}^{m} a_{j}^{m}+B_{s, q, j}^{m} b_{j}^{m}+C_{s, q, j}^{m} c_{j}^{m}\right] \\
& +\sum_{j=3}^{4}\left[A_{s, q, j}^{m} \alpha_{j}^{m}+B_{s, q, j}^{m} \beta_{j}^{m}+\Gamma_{s, q, j}^{m} \gamma_{j}^{m}\right. \\
& \left.+\Delta_{s, q, j}^{m} \delta_{j}^{m}+E_{s, q, j}^{m} \varepsilon_{j}^{m}+Z_{s, q, j}^{m} \zeta_{j}^{m}\right]=0
\end{aligned}
$$

for $s=1,2 ; q=1,2, . ., 9$.

The parameter $s$ refers to the enumeration of the handled boundary condition. For symbolism convenience, the value $s=1$ corresponds to Eq.(41) while $s=$ 2 is assigned to Eq.(40). The parameter $q$ denotes the cylindrical harmonic 
dyad on which projection has been performed. The sequence of projections follows exactly the series of appearance of the harmonic dyadics in Eqs. $(37,38)$. Every subsystem of the form (43) consists of 18 equations with 18 unknowns. The specific form of the coefficients of every subsystem is the outcome of the mentioned projection process and all these terms are given in the Appendix.

The crucial remark is that every system of the form (43) is homogeneous as was expected in the framework of wave propagation investigation. So the determinant of the matrix of the coefficients of every subsystem must vanish and this is the fundamental relation for connecting the propagation phase parameters $\omega$ and $p$ and building the dispersion relation for every particular propagation mode.

Every one of these dispersion relations refers to a specific mode and just coincides with the transcendental equation - connecting $\omega$ and $p$ - produced after setting to zero the determinant of the $18 \times 18$ matrix corresponding to a specific azimouthal value $m$. After the establishment of the curve $p=f_{m}(\omega)$, the corresponding propagating mode is constructed by solving the system (43), i.e. by determining the involved unknown coefficients naturally modulo a multiplicative constant, expressing simply the amplitude of the mode.

\section{$5 \quad$ Summary and Conclusions}

In this study, we presented a three dimensional (3D) analytical model of wave propagation in cylindrical waveguides exhibiting microstructure. The application motivation was to generate a three dimensional framework for the investigation of elastic wave propagation in long cortical bones. The first outcome of the present work was the spectral decomposition of the boundary value problem emerging via wave propagation analysis. Starting from the general Midlin's Form II gradient elasticity theory, we developed the spectral representation of the specific gradient elasticity problem and constructed all the possible propagating modes in cylindrical geometry. Two main byproducts arose which constitute generalizations of well known important features of classical elasticity and which are indispensable for modeling the gradient elasticity problem, while independently possessing their own theoretical value. First we accomplished the construction of the set of dyadic Navier eigenfunctions which constitute the generalization of the Navier eigenvectors. This set is a basis in the space of solutions of gradient elasticity in cylindrical coordinates. The restriction of the Navier eigendyadics on cylindrical surfaces gives birth to the dyadic cylindrical harmonics, which constitute the generalization of the well known vector harmonics. This set is also a basis in the sense that the trace of every dyadic field on a cylindrical surface can be represented as a countable superposition of dyadic cylindrical harmonics. The outcome of the current analysis aims at constructing the analytical dispersion curves of guided waves propagating through cortical bones. The numerical treatment of the problem is an independent and demanding computational task, which after completion, is expected to be highly useful for the assessment of pathological situations of bone and in bone healing 
evaluation.

\section{Acknowledgment}

We would like to thank Dr. Maria Vavva for helpful discussions.

\section{Appendix}

\section{A The structure of the traveling modes subsys- tems}

The elements of the subsystems as well as of the matrices whose determinants represent the dispersion relations are presented herein. It is very useful to introduce here some symbolic nomeclature. More precisely, we note that it is useful to represent these coefficients - and other involved auxiliary quantities as functions of the polar distance, after restriction to $r=a$. For example, $A_{1,1, j}^{m}$ stands for $A_{1,1, j}^{m}(a)$, where the function $A_{1,1, j}^{m}(r)$ is easily obtained from the relevant definition of $A_{1,1, j}^{m}$. In addition $B_{1,1, j}^{\prime m}$, for instance, denotes $B_{1,1, j}^{\prime m}(a)$, i.e. $\left.\frac{d}{d r} B_{1,1, j}^{m}(r)\right|_{r=a}$. This terminology simplifies the expression of the results extensively.

1. The coefficients offered by boundary condition (41).

1.1. Projection on $\hat{\mathbf{r}} \mathbf{P}_{m}$

$$
A_{1,1, j}^{m}=-\left[2 \alpha_{1}+\alpha_{2}+2 \alpha_{3}\right] k_{j}^{2} \ddot{J}_{m}\left(\xi_{j} a\right)+2\left[\alpha_{4}+\alpha_{5}\right] J_{m}^{(i v)}\left(\xi_{j} a\right), j=1,2
$$

(mention that $A_{1,1, j}^{m}(r)=-\left[2 \alpha_{1}+\alpha_{2}+2 \alpha_{3}\right] k_{j}^{2} \ddot{J}_{m}\left(\xi_{j} r\right)+2\left[\alpha_{4}+\alpha_{5}\right] J_{m}^{(i v)}\left(\xi_{j} r\right)$ )

$$
\begin{aligned}
& B_{1,1, j}^{m}=-\left.\left[2 \alpha_{1}+\alpha_{2}+2 \alpha_{3}\right] k_{j}^{2} i m \frac{d}{d\left(\xi_{j} r\right)}\left(\frac{J_{m}\left(\xi_{j} r\right)}{\left(\xi_{j} r\right)}\right)\right|_{r=a}+ \\
& \left.2\left[\alpha_{4}+\alpha_{5}\right] \xi_{j}^{2} i m \frac{d^{3}}{d\left(\xi_{j} r\right)^{3}}\left(\frac{J_{m}\left(\xi_{j} r\right)}{\left(\xi_{j} r\right)}\right)\right|_{r=a}, j=1,2 \\
& C_{1,1, j}^{m}=\left[2 \alpha_{1}+\alpha_{2}+2 \alpha_{3}\right] \frac{p^{2}}{\xi_{j}^{2}} k_{j}^{2} \ddot{J}_{m}\left(\xi_{j} a\right)-2\left[\alpha_{4}+\alpha_{5}\right] p^{2} J_{m}^{(i v)}\left(\xi_{j} a\right)
\end{aligned}
$$




$$
\begin{aligned}
& A_{1,1, j}^{m}=-\frac{1}{2}\left[\alpha_{1}+2 \alpha_{3}\right] k_{j}^{2}\left(\ddot{J}_{m}\left(\xi_{j} a\right)+\frac{k_{j}^{2}}{\xi_{j}^{2}} J_{m}\left(\xi_{j} a\right)\right) \\
& +2\left[\alpha_{4}+\alpha_{5}\right] \xi_{j}^{2}\left(J_{m}^{(i v)}\left(\xi_{j} a\right)+\frac{k_{j}^{2}}{\xi_{j}^{2}} \ddot{J}_{m}\left(\xi_{j} a\right)\right), j=3,4 \\
& B_{1,1, j}^{m}=-\left.\frac{1}{2}\left[\alpha_{1}+2 \alpha_{3}\right] k_{j}^{2} i m \frac{d}{d\left(\xi_{j} r\right)}\left(\frac{J_{m}\left(\xi_{j} r\right)}{\left(\xi_{j} r\right)}\right)\right|_{r=a} \\
& +\left.2\left[\alpha_{4}+\alpha_{5}\right] \xi_{j}^{2} i m \frac{d^{3}}{d\left(\xi_{j} r\right)^{3}}\left(\frac{J_{m}\left(\xi_{j} r\right)}{\left(\xi_{j} r\right)}\right)\right|_{r=a}, j=3,4 \\
& \Gamma_{1,1, j}^{m}=\frac{1}{2}\left[\alpha_{1}+2 \alpha_{3}\right] k_{j}^{2} \frac{p^{2}}{\xi_{j}^{2}} \ddot{J}_{m}\left(\xi_{j} a\right)-2\left[\alpha_{4}+\alpha_{5}\right] p^{2} J_{m}^{(i v)}\left(\xi_{j} a\right) \\
& \Delta_{1,1, j}^{m}=-\left.\frac{1}{2}\left[\alpha_{1}+2 \alpha_{3}\right] k_{j}^{2} i m \frac{d}{d\left(\xi_{j} r\right)}\left(\frac{J_{m}\left(\xi_{j} r\right)}{\left(\xi_{j} r\right)}\right)\right|_{r=a} \\
& +\left.2\left[\alpha_{4}+\alpha_{5}\right] \xi_{j}^{2} i m \frac{d^{3}}{d\left(\xi_{j} r\right)^{3}}\left(\frac{J_{m}\left(\xi_{j} r\right)}{\left(\xi_{j} r\right)}\right)\right|_{r=a} \\
& E_{1,1, j}^{m}=-\frac{1}{2}\left[\alpha_{1}+2 \alpha_{3}\right] k_{j}^{2}\left(\frac{\dot{J}_{m}\left(\xi_{j} a\right)}{\xi_{j} a}-\frac{m^{2}}{\left(\xi_{j} a\right)^{2}} J_{m}\left(\xi_{j} a\right)\right) \\
& +\left.2\left[\alpha_{4}+\alpha_{5}\right] \xi_{j}^{2} \frac{d^{2}}{d\left(\xi_{j} r\right)^{2}}\left(\frac{\dot{J}_{m}\left(\xi_{j} r\right)}{\xi_{j} r}-\frac{m^{2}}{\left(\xi_{j} r\right)^{2}} J_{m}\left(\xi_{j} r\right)\right)\right|_{r=a} \\
& Z_{1,1, j}^{m}=\left.\frac{1}{2}\left[\alpha_{1}+2 \alpha_{3}\right] k_{j}^{2} \frac{p^{2}}{\xi_{j}^{2}} i m \frac{d}{d\left(\xi_{j} r\right)}\left(\frac{J_{m}\left(\xi_{j} r\right)}{\left(\xi_{j} r\right)}\right)\right|_{r=a} \\
& -\left.2\left[\alpha_{4}+\alpha_{5}\right] p^{2} i m \frac{d^{3}}{d\left(\xi_{j} r\right)^{3}}\left(\frac{J_{m}\left(\xi_{j} r\right)}{\left(\xi_{j} r\right)}\right)\right|_{r=a}
\end{aligned}
$$

1.2. Projection on $\left(\hat{\mathbf{r}} \mathbf{B}_{m}\right)_{s}$

$$
\begin{aligned}
& A_{1,2, j}^{m}=-\left.\frac{1}{2}\left[5 \alpha_{1}+2 \alpha_{2}+6 \alpha_{3}\right] k_{j}^{2} i m \frac{d}{d\left(\xi_{j} r\right)}\left(\frac{J_{m}\left(\xi_{j} r\right)}{\left(\xi_{j} r\right)}\right)\right|_{r=a} \\
& +\left.4\left[\alpha_{4}+\alpha_{5}\right] \xi_{j}^{2} i m \frac{d^{3}}{d\left(\xi_{j} r\right)^{3}}\left(\frac{J_{m}\left(\xi_{j} r\right)}{\left(\xi_{j} r\right)}\right)\right|_{r=a}, j=1,2 \\
& B_{1,2, j}^{m}=\left[2 \alpha_{1}+\alpha_{2}+2 \alpha_{3}\right] k_{j}^{2} \ddot{J}_{m}\left(\xi_{j} a\right) \\
& -\frac{1}{2}\left[\alpha_{1}+2 \alpha_{3}\right] k_{j}^{2}\left(\frac{\dot{J}_{m}\left(\xi_{j} a\right)}{\xi_{j} a}-\frac{m^{2}}{\left(\xi_{j} a\right)^{2}} J_{m}\left(\xi_{j} a\right)\right) \\
& +2\left[\alpha_{4}+\alpha_{5}\right] \xi_{j}^{2}\left[\left.2 \frac{d^{2}}{d\left(\xi_{j} r\right)^{2}}\left(\frac{\dot{J}_{m}\left(\xi_{j} r\right)}{\xi_{j} r}-\frac{m^{2}}{\left(\xi_{j} r\right)^{2}} J_{m}\left(\xi_{j} r\right)\right)\right|_{r=a}\right. \\
& \left.+\ddot{J}_{m}\left(\xi_{j} a\right)\right], \quad j=1,2
\end{aligned}
$$




$$
\begin{aligned}
& C_{1,2, j}^{m}=\left.\frac{1}{2}\left[5 \alpha_{1}+2 \alpha_{2}+6 \alpha_{3}\right] k_{j}^{2} \frac{p^{2}}{\xi_{j}^{2}} i m \frac{d}{d\left(\xi_{j} r\right)}\left(\frac{J_{m}\left(\xi_{j} r\right)}{\left(\xi_{j} r\right)}\right)\right|_{r=a} \\
& -\left.4\left[\alpha_{4}+\alpha_{5}\right] p^{2} i m \frac{d^{3}}{d\left(\xi_{j} r\right)^{3}}\left(\frac{J_{m}\left(\xi_{j} r\right)}{\left(\xi_{j} r\right)}\right)\right|_{r=a} \\
& A_{1,2, j}^{m}=-\left.\frac{1}{2}\left[\alpha_{1}+3 \alpha_{3}-2 \alpha_{4}-3 \alpha_{5}\right] k_{j}^{2} i m \frac{d}{d\left(\xi_{j} r\right)}\left(\frac{J_{m}\left(\xi_{j} r\right)}{\left(\xi_{j} r\right)}\right)\right|_{r=a} \\
& +\left.4\left[\alpha_{4}+\alpha_{5}\right] \xi_{j}^{2} i m \frac{d^{3}}{d\left(\xi_{j} r\right)^{3}}\left(\frac{J_{m}\left(\xi_{j} r\right)}{\left(\xi_{j} r\right)}\right)\right|_{r=a}, j=3,4 \\
& B_{1,2, j}^{m}=-\frac{1}{2}\left[\alpha_{1}+3 \alpha_{3}\right] k_{j}^{2}\left(\frac{\dot{J}_{m}\left(\xi_{j} a\right)}{\xi_{j} a}-\frac{m^{2}}{\left(\xi_{j} a\right)^{2}} J_{m}\left(\xi_{j} a\right)\right)-\frac{1}{2} \alpha_{3} k_{j}^{2} J_{m}\left(\xi_{j} a\right) \\
& +2\left[\alpha_{4}+\alpha_{5}\right] \xi_{j}^{2}\left[\left.2 \frac{d^{2}}{d\left(\xi_{j} r\right)^{2}}\left(\frac{\dot{J}_{m}\left(\xi_{j} r\right)}{\xi_{j} r}-\frac{m^{2}}{\left(\xi_{j} r\right)^{2}} J_{m}\left(\xi_{j} r\right)\right)\right|_{r=a}\right. \\
& \left.+\ddot{J}_{m}\left(\xi_{j} a\right)\right]-\frac{1}{2}\left[2 \alpha_{4}+3 \alpha_{5}\right] k_{j}^{2} \ddot{J}_{m}\left(\xi_{j} a\right), j=3,4 \\
& \Gamma_{1,2, j}^{m}=\left.\frac{1}{2}\left[\alpha_{1}+3 \alpha_{3}-2 \alpha_{4}-3 \alpha_{5}\right] k_{j}^{2} \frac{p^{2}}{\xi_{j}^{2}} i m \frac{d}{d\left(\xi_{j} r\right)}\left(\frac{J_{m}\left(\xi_{j} r\right)}{\left(\xi_{j} r\right)}\right)\right|_{r=a} \\
& -\left.4\left[\alpha_{4}+\alpha_{5}\right] p^{2} i m \frac{d^{3}}{d\left(\xi_{j} r\right)^{3}}\left(\frac{J_{m}\left(\xi_{j} r\right)}{\left(\xi_{j} r\right)}\right)\right|_{r=a} \\
& \Delta_{1,2, j}^{m}=-\frac{1}{2}\left[\alpha_{1}+3 \alpha_{3}\right] k_{j}^{2}\left(\frac{\dot{J}_{m}\left(\xi_{j} a\right)}{\xi_{j} a}-\frac{m^{2}}{\left(\xi_{j} a\right)^{2}} J_{m}\left(\xi_{j} a\right)\right)-\frac{1}{2} \alpha_{3} k_{j}^{2} J_{m}\left(\xi_{j} a\right) \\
& +2\left[\alpha_{4}+\alpha_{5}\right] \xi_{j}^{2}\left[\left.2 \frac{d^{2}}{d\left(\xi_{j} r\right)^{2}}\left(\frac{\dot{J}_{m}\left(\xi_{j} r\right)}{\xi_{j} r}-\frac{m^{2}}{\left(\xi_{j} r\right)^{2}} J_{m}\left(\xi_{j} r\right)\right)\right|_{r=a}\right. \\
& \left.+\ddot{J}_{m}\left(\xi_{j} a\right)\right]-\frac{1}{2}\left[2 \alpha_{4}+3 \alpha_{5}\right] k_{j}^{2} \ddot{J}_{m}\left(\xi_{j} a\right) \\
& E_{1,2, j}^{m}=\left.\frac{1}{2}\left[\alpha_{1}+3 \alpha_{3}-2 \alpha_{4}-3 \alpha_{5}\right] k_{j}^{2} i m \frac{d}{d\left(\xi_{j} r\right)}\left(\frac{J_{m}\left(\xi_{j} r\right)}{\left(\xi_{j} r\right)}\right)\right|_{r=a} \\
& -\left.4\left[\alpha_{4}+\alpha_{5}\right] \xi_{j}^{2} i m \frac{d^{3}}{d\left(\xi_{j} r\right)^{3}}\left(\frac{J_{m}\left(\xi_{j} r\right)}{\left(\xi_{j} r\right)}\right)\right|_{r=a}
\end{aligned}
$$




$$
\begin{aligned}
& Z_{1,2, j}^{m}=\frac{1}{2}\left[\alpha_{1}+3 \alpha_{3}\right] k_{j}^{2} \frac{p^{2}}{\xi_{j}^{2}}\left(\frac{\dot{J}_{m}\left(\xi_{j} a\right)}{\xi_{j} a}-\frac{m^{2}}{\left(\xi_{j} a\right)^{2}} J_{m}\left(\xi_{j} a\right)\right) \\
& +\frac{1}{2} \alpha_{3} k_{j}^{2} \frac{p^{2}}{\xi_{j}^{2}} J_{m}\left(\xi_{j} a\right) \\
& -2\left[\alpha_{4}+\alpha_{5}\right] p^{2}\left[\left.2 \frac{d^{2}}{d\left(\xi_{j} r\right)^{2}}\left(\frac{\dot{J}_{m}\left(\xi_{j} r\right)}{\xi_{j} r}-\frac{m^{2}}{\left(\xi_{j} r\right)^{2}} J_{m}\left(\xi_{j} r\right)\right)\right|_{r=a}\right. \\
& \left.+\ddot{J}_{m}\left(\xi_{j} a\right)\right]+\frac{1}{2}\left[2 \alpha_{4}+3 \alpha_{5}\right] k_{j}^{2} \frac{p^{2}}{\xi_{j}^{2}} \ddot{J}_{m}\left(\xi_{j} a\right)
\end{aligned}
$$

1.3. Projection on $\hat{\phi} \mathbf{B}_{m}$

$$
\begin{gathered}
A_{1,3, j}^{m}=-\frac{1}{2}\left[\alpha_{1}+2 \alpha_{3}\right] k_{j}^{2}\left(\frac{\dot{J}_{m}\left(\xi_{j} a\right)}{\xi_{j} a}-\frac{m^{2}}{\left(\xi_{j} a\right)^{2}} J_{m}\left(\xi_{j} a\right)\right) \\
+\left.2\left[\alpha_{4}+\alpha_{5}\right] \xi_{j}^{2} \frac{d^{2}}{d\left(\xi_{j} r\right)^{2}}\left(\frac{\dot{J}_{m}\left(\xi_{j} r\right)}{\xi_{j} r}-\frac{m^{2}}{\left(\xi_{j} r\right)^{2}} J_{m}\left(\xi_{j} r\right)\right)\right|_{r=a}, \quad j=1 \\
B_{1,3, j}^{m}=\left.\frac{1}{2}\left[\alpha_{1}+2 \alpha_{3}\right] k_{j}^{2} i m \frac{d}{d\left(\xi_{j} r\right)}\left(\frac{J_{m}\left(\xi_{j} r\right)}{\left(\xi_{j} r\right)}\right)\right|_{r=a} \\
-\left.2\left[\alpha_{4}+\alpha_{5}\right] \xi_{j}^{2} \frac{d^{3}}{d\left(\xi_{j} r\right)^{3}}\left(\frac{J_{m}\left(\xi_{j} r\right)}{\left(\xi_{j} r\right)}\right)\right|_{r=a}, \quad j=1,2 \\
C_{1,3, j}^{m}=\frac{1}{2}\left[\alpha_{1}+2 \alpha_{3}\right] k_{j}^{2} \frac{p^{2}}{\xi_{j}^{2}}\left(\frac{\dot{J}_{m}\left(\xi_{j} a\right)}{\xi_{j} a}-\frac{m^{2}}{\left(\xi_{j} a\right)^{2}} J_{m}\left(\xi_{j} a\right)\right) \\
-\left.2\left[\alpha_{4}+\alpha_{5}\right] p^{2} \frac{d^{2}}{d\left(\xi_{j} r\right)^{2}}\left(\frac{\dot{J}_{m}\left(\xi_{j} r\right)}{\xi_{j} r}-\frac{m^{2}}{\left(\xi_{j} r\right)^{2}} J_{m}\left(\xi_{j} r\right)\right)\right|_{r=a} \\
+\frac{1}{2}\left[\alpha_{4}+3 \alpha_{5}\right] k_{j}^{2} \ddot{J_{m}\left(\xi_{j} a\right), j}=3,4 \\
A_{1,3, j}^{m}=-\frac{1}{2} \alpha_{3} k_{j}^{2}\left(\frac{\dot{J}_{m}\left(\xi_{j} a\right)}{\xi_{j} a}+\left(\frac{k_{j}^{2}}{\xi_{j}^{2}}-\frac{m^{2}}{\left(\xi_{j} a\right)^{2}}\right) J_{m}\left(\xi_{j} a\right)\right) \\
\left.\xi_{j}\left[\alpha_{4}+\alpha_{5}\right] \xi_{j}^{2} \frac{d^{2}}{d\left(\xi_{j} r\right)^{2}}\left(\frac{\dot{J}_{m}\left(\xi_{j} r\right)}{\xi_{j} r}+\left(\frac{k_{j}^{2}}{\xi_{j}^{2}}-\frac{m^{2}}{\left(\xi_{j} r\right)^{2}}\right) J_{m}\left(\xi_{j} r\right)\right)\right|_{r=a}
\end{gathered}
$$

20 


$$
\begin{gathered}
B_{1,3, j}^{m}=\left.\frac{1}{2}\left[\alpha_{3}-2 \alpha_{4}-3 \alpha_{5}\right] k_{j}^{2} i m \frac{d}{d\left(\xi_{j} r\right)}\left(\frac{J_{m}\left(\xi_{j} r\right)}{\left(\xi_{j} r\right)}\right)\right|_{r=a} \\
-\left.2\left[\alpha_{4}+\alpha_{5}\right] \xi_{j}^{2} i m \frac{d^{3}}{d\left(\xi_{j} r\right)^{3}}\left(\frac{J_{m}\left(\xi_{j} r\right)}{\left(\xi_{j} r\right)}\right)\right|_{r=a}, j=3,4 \\
\Gamma_{1,3, j}^{m}=\frac{1}{2} \alpha_{3} k_{j}^{2} \frac{p^{2}}{\xi_{j}^{2}}\left(\frac{\dot{J}_{m}\left(\xi_{j} a\right)}{\xi_{j} a}-\frac{m^{2}}{\left(\xi_{j} a\right)^{2}} J_{m}\left(\xi_{j} a\right)\right) \\
-\left.2\left[\alpha_{4}+\alpha_{5}\right] p^{2} \frac{d^{2}}{d\left(\xi_{j} r\right)^{2}}\left(\frac{\dot{J}_{m}\left(\xi_{j} r\right)}{\xi_{j} r}-\frac{m^{2}}{\left(\xi_{j} r\right)^{2}} J_{m}\left(\xi_{j} r\right)\right)\right|_{r=a} \\
+\frac{1}{2}\left[2 \alpha_{4}+3 \alpha_{5}\right] k_{j}^{2} \frac{p^{2}}{\xi_{j}^{2}} \ddot{J}_{m}\left(\xi_{j} a\right) \\
\Delta_{1,3, j}^{m}=\left.\frac{1}{2}\left[\alpha_{3}-2 \alpha_{4}-3 \alpha_{5}\right] k_{j}^{2} i m \frac{d}{d\left(\xi_{j} r\right)}\left(\frac{J_{m}\left(\xi_{j} r\right)}{\left(\xi_{j} r\right)}\right)\right|_{r=a} \\
\quad+\left.2\left[\alpha_{4}+\alpha_{5}\right] \xi_{j}^{2} i m \frac{d^{3}}{d\left(\xi_{j} r\right)^{3}}\left(\frac{J_{m}\left(\xi_{j} r\right)}{\left(\xi_{j} r\right)}\right)\right|_{r=a} \\
E_{1,3, j}^{m}=-\frac{1}{2}\left[\alpha_{3}-2 \alpha_{4}-3 \alpha_{5}\right] k_{j}^{2} \ddot{J}_{m}\left(\xi_{j} a\right)+2\left[\alpha_{4}+\alpha_{5}\right] \xi_{j}^{2} J_{m}^{(i v)}\left(\xi_{j} a\right) \\
Z_{1,3, j}^{m}=-\left.\frac{1}{2}\left[\alpha_{3}-2 \alpha_{4}-3 \alpha_{5}\right] k_{j}^{2} \frac{p^{2}}{\xi_{j}^{2}} i m \frac{d}{d\left(\xi_{j} r\right)}\left(\frac{d_{m}\left(\xi_{j} r\right)}{\left(\xi_{j} r\right)}\right)\right|_{r=a} \\
\left.\left(\frac{J_{m}\left(\xi_{j} r\right)}{\left(\xi_{j} r\right)}\right)\right|_{r=a}
\end{gathered}
$$

1.4. Projection on $\left(\hat{\mathbf{r}} \mathbf{C}_{m}\right)_{s}$

$$
\begin{gathered}
A_{1,4, j}^{m}=-\frac{1}{2}\left[5 \alpha_{1}+2 \alpha_{2}+6 \alpha_{3}\right] k_{j}^{2} \frac{p}{\xi_{j}} \dot{J}_{m}\left(\xi_{j} a\right)+4\left[\alpha_{4}+\alpha_{5}\right] p \xi_{j} J_{m}^{(i i i)}\left(\xi_{j} a\right) \\
j=1,2 \\
B_{1,4, j}^{m}=-\frac{1}{2}\left[\alpha_{1}+2 \alpha_{3}\right] k_{j}^{2} \frac{p}{\xi_{j}} i m \frac{J_{m}\left(\xi_{j} a\right)}{\left(\xi_{j} a\right)} \\
+\left.2\left[\alpha_{4}+\alpha_{5}\right] p \xi_{j} i m \frac{d^{2}}{d\left(\xi_{j} r\right)^{2}}\left(\frac{J_{m}\left(\xi_{j} r\right)}{\left(\xi_{j} r\right)}\right)\right|_{r=a} \cdot j=1,2
\end{gathered}
$$

$$
C_{1,4, j}^{m}=-\frac{1}{2}\left[3 \alpha_{1}+2 \alpha_{2}+2 \alpha_{3}\right] k_{j}^{2} \frac{p}{\xi_{j}} \dot{J}_{m}\left(\xi_{j} a\right)-\frac{1}{2}\left[\alpha_{1}+2 \alpha_{3}\right] k_{j}^{2} \frac{p}{\xi_{j}}\left(1-\frac{p^{2}}{\xi_{j}^{2}}\right) \dot{J}_{m}\left(\xi_{j} a\right)
$$$$
+2\left[\alpha_{4}+\alpha_{5}\right] p \xi_{j}\left(1-\frac{p^{2}}{\xi_{j}^{2}}\right) J_{m}^{(i i i)}\left(\xi_{j} a\right)
$$ 


$$
\begin{gathered}
A_{1,4, j}^{m}=-\frac{1}{2}\left[\alpha_{1}+3 \alpha_{3}-2 \alpha_{4}-3 \alpha_{5}\right] k_{j}^{2} \frac{p}{\xi_{j}} \dot{J}_{m}\left(\xi_{j} a\right)+4\left[\alpha_{4}+\alpha_{5}\right] p \xi_{j} J_{m}^{(i i i)}\left(\xi_{j} a\right) \\
j=3,(\mathrm{~A} .31) \\
B_{1,4, j}^{m}=-\frac{1}{2}\left[\alpha_{1}+2 \alpha_{3}\right] k_{j}^{2} \frac{p}{\xi_{j}} i m \frac{J_{m}\left(\xi_{j} a\right)}{\left(\xi_{j} a\right)} \\
+\left.2\left[\alpha_{4}+\alpha_{5}\right] p \xi_{j} i m \frac{d^{2}}{d\left(\xi_{j} r\right)^{2}}\left(\frac{J_{m}\left(\xi_{j} r\right)}{\left(\xi_{j} r\right)}\right)\right|_{r=a}, j=3,4 \\
\Gamma_{1,4, j}^{m}=\frac{1}{2}\left[\alpha_{1}+\alpha_{3}\right] k_{j}^{2} \frac{p^{3}}{\xi_{j}^{3}} \dot{J}_{m}\left(\xi_{j} a\right)-\frac{1}{2} \alpha_{3} k_{j}^{2} \frac{p}{\xi_{j}}\left(1-\frac{p^{2}}{\xi_{j}^{2}}\right) \dot{J}_{m}\left(\xi_{j} a\right) \\
+2\left[\alpha_{4}+\alpha_{5}\right] p \xi_{j}\left(1-\frac{p^{2}}{\xi_{j}^{2}}\right) J_{m}^{(i i i)}\left(\xi_{j} a\right)+\frac{1}{2}\left[2 \alpha_{4}+3 \alpha_{5}\right] k_{j}^{2} \frac{p}{\xi_{j}} \dot{J}_{m}\left(\xi_{j} a\right)(\mathrm{A} .33) \\
\Delta_{1,4, j}^{m}=-\frac{1}{2}\left[\alpha_{1}+2 \alpha_{3}\right] k_{j}^{2} \frac{p}{\xi_{j}} i m \frac{J_{m}\left(\xi_{j} a\right)}{\left(\xi_{j} a\right)} \\
+\left.2\left[\alpha_{4}+\alpha_{5}\right] p \xi_{j} i m \frac{d^{2}}{d\left(\xi_{j} r\right)^{2}}\left(\frac{J_{m}\left(\xi_{j} r\right)}{\left(\xi_{j} r\right)}\right)\right|_{r=a} \\
E_{1,4, j}^{m}=0 \\
Z_{1,4, j}^{m}=-\frac{1}{2}\left[\alpha_{1}+2 \alpha_{3}\right] k_{j}^{2} \frac{p}{\xi_{j}} i m \frac{J_{m}\left(\xi_{j} a\right)}{\left(\xi_{j} a\right)} \\
+\left.2\left[\alpha_{4}+\alpha_{5}\right] p \xi_{j} \frac{d^{2}}{d\left(\xi_{j} r\right)^{2}}\left(\frac{J_{m}\left(\xi_{j} r\right)}{\left(\xi_{j} r\right)}\right)\right|_{r=a}
\end{gathered}
$$

1.5. Projection on $\left(\hat{\phi} \mathbf{C}_{m}\right)_{s}$

$$
\begin{gathered}
A_{1,5, j}^{m}=-\left[\alpha_{1}+2 \alpha_{3}\right] k_{j}^{2} \frac{p}{\xi_{j}} i m \frac{J_{m}\left(\xi_{j} a\right)}{\left(\xi_{j} a\right)} \\
+\left.4\left[\alpha_{4}+\alpha_{5}\right] p \xi_{j} i m \frac{d^{2}}{d\left(\xi_{j} r\right)^{2}}\left(\frac{J_{m}\left(\xi_{j} r\right)}{\left(\xi_{j} r\right)}\right)\right|_{r=a}, j=1,2 \\
B_{1,5, j}^{m}=\frac{1}{2}\left[\alpha_{1}+2 \alpha_{3}\right] k_{j}^{2} \frac{p}{\xi_{j}} \dot{J}_{m}\left(\xi_{j} a\right)-2\left[\alpha_{4}+\alpha_{5}\right] p \xi_{j} J_{m}^{(i i i)}\left(\xi_{j} a\right), j=1,2 \\
C_{1,5, j}^{m}=-\frac{1}{2}\left[\alpha_{1}+2 \alpha_{3}\right] k_{j}^{2} \frac{p}{\xi_{j}}\left(1-\frac{p^{2}}{\xi_{j}^{2}}\right) i m \frac{J_{m}\left(\xi_{j} a\right)}{\left(\xi_{j} a\right)} \\
+\left.2\left[\alpha_{4}+\alpha_{5}\right] p \xi_{j}\left(1-\frac{p^{2}}{\xi_{j}^{2}}\right) i m \frac{d^{2}}{d\left(\xi_{j} r\right)^{2}}\left(\frac{J_{m}\left(\xi_{j} r\right)}{\left(\xi_{j} r\right)}\right)\right|_{r=a}
\end{gathered}
$$




$$
\begin{gathered}
A_{1,5, j}^{m}=-\alpha_{3} k_{j}^{2} \frac{p}{\xi_{j}} i m \frac{J_{m}\left(\xi_{j} a\right)}{\left(\xi_{j} a\right)} \\
+\left.4\left[\alpha_{4}+\alpha_{5}\right] p \xi_{j} i m \frac{d^{2}}{d\left(\xi_{j} r\right)^{2}}\left(\frac{J_{m}\left(\xi_{j} r\right)}{\left(\xi_{j} r\right)}\right)\right|_{r=a}, j=3,4 \\
B_{1,5, j}^{m}=\frac{1}{2} \alpha_{3} k_{j}^{2} \frac{p}{\xi_{j}} \dot{J}_{m}\left(\xi_{j} a\right)-2\left[\alpha_{4}+\alpha_{5}\right] p \xi_{j} J_{m}^{(i i i)}\left(\xi_{j} a\right), j=3,4 \\
\Gamma_{1,5, j}^{m}=-\frac{1}{2} \alpha_{3} k_{j}^{2} \frac{p}{\xi_{j}}\left(1-\frac{p^{2}}{\xi_{j}^{2}}\right) i m \frac{J_{m}\left(\xi_{j} a\right)}{\left(\xi_{j} a\right)} \\
+\left.2\left[\alpha_{4}+\alpha_{5}\right] p \xi_{j}\left(1-\frac{p^{2}}{\xi_{j}^{2}}\right) i m \frac{d^{2}}{d\left(\xi_{j} r\right)^{2}}\left(\frac{J_{m}\left(\xi_{j} r\right)}{\left(\xi_{j} r\right)}\right)\right|_{r=a} \\
\Delta_{1,5, j}^{m}=\frac{1}{2}\left[\alpha_{3}-2 \alpha_{4}-3 \alpha_{5}\right] k_{j}^{2} \frac{p}{\xi_{j}} \dot{J}_{m}\left(\xi_{j} a\right)-2\left[\alpha_{4}+\alpha_{5}\right] p \xi_{j} J_{m}^{(i i i)}\left(\xi_{j} a\right) \\
E_{1,5, j}^{m}=0, \quad Z_{1,5, j}^{m}=\Delta_{1,5, j}^{m}
\end{gathered}
$$

1.6. Projection on $-i \hat{\mathbf{z}} \mathbf{C}_{m}$

$$
\begin{gathered}
A_{1,6, j}^{m}=\frac{1}{2}\left[\alpha_{1}+2 \alpha_{3}\right] k_{j}^{2} \frac{p^{2}}{\xi_{j}^{2}} J_{m}\left(\xi_{j} a\right)-2\left[\alpha_{4}+\alpha_{5}\right] p^{2} \ddot{J}_{m}\left(\xi_{j} a\right), j=1,2 \\
B_{1,6, j}^{m}=0, \quad C_{1,6, j}^{m}=A_{1,6, j}^{m}, j=1,2 \\
A_{1,6, j}^{m}=-\frac{1}{2} \alpha_{3} k_{j}^{2} J_{m}\left(\xi_{j} a\right)+2\left[\alpha_{4}+\alpha_{5}\right] \xi_{j}^{2} \ddot{J}_{m}\left(\xi_{j} a\right) \\
-\frac{1}{2}\left[2 \alpha_{4}+3 \alpha_{5}\right] k_{j}^{2} \ddot{J}_{m}\left(\xi_{j} a\right), j=3,4 \\
B_{1,6, j}^{m}=-\left.\frac{1}{2}\left[2 \alpha_{4}+3 \alpha_{5}\right] k_{j}^{2} i m \frac{d}{d\left(\xi_{j} r\right)}\left(\frac{J_{m}\left(\xi_{j} r\right)}{\left(\xi_{j} r\right)}\right)\right|_{r=a}, j=3,4 \\
C_{1,6, j}^{m}=-\frac{p^{2}}{\xi_{j}^{2}} A_{1,6, j}^{m}, j=3,4 \\
\Delta_{1,6, j}^{m}=E_{1,6, j}^{m}=Z_{1,6, j}^{m}=0, \quad j=3,4
\end{gathered}
$$

1.7. Projection on $\left(\hat{\mathbf{r}} \mathbf{B}_{m}\right)_{\alpha}$ 


$$
\begin{aligned}
& A_{1,7, j}^{m}=-\left.\frac{1}{2}\left[3 \alpha_{1}+2 \alpha_{2}+2 \alpha_{3}\right] k_{j}^{2} i m \frac{d}{d\left(\xi_{j} r\right)}\left(\frac{J_{m}\left(\xi_{j} r\right)}{\left(\xi_{j} r\right)}\right)\right|_{r=a}, j=1,2 \\
& B_{1,7, j}^{m}=\frac{1}{2}\left[3 \alpha_{1}+2 \alpha_{2}+2 \alpha_{3}\right] k_{j}^{2} \ddot{J}_{m}\left(\xi_{j} a\right)-\frac{1}{2}\left[\alpha_{1}+2 \alpha_{3}\right] k_{j}^{2} J_{m}\left(\xi_{j} a\right) \\
& +2\left[\alpha_{4}+\alpha_{5}\right] \xi_{j}^{2} \ddot{J}_{m}\left(\xi_{j} a\right), j=1,2 \\
& C_{1,7, j}^{m}=\left.\frac{1}{2}\left[3 \alpha_{1}+2 \alpha_{2}+2 \alpha_{3}\right] k_{j}^{2} \frac{p^{2}}{\xi_{j}^{2}} i m \frac{d}{d\left(\xi_{j} r\right)}\left(\frac{J_{m}\left(\xi_{j} r\right)}{\left(\xi_{j} r\right)}\right)\right|_{r=a} \\
& A_{1,7, j}^{m}=-\left.\frac{1}{2}\left[\alpha_{1}+\alpha_{3}+2 \alpha_{4}+3 \alpha_{5}\right] k_{j}^{2} i m \frac{d}{d\left(\xi_{j} r\right)}\left(\frac{J_{m}\left(\xi_{j} r\right)}{\left(\xi_{j} r\right)}\right)\right|_{r=a}, j=3, \text { 4A.54) } \\
& B_{1,7, j}^{m}=-\frac{1}{2}\left[\alpha_{1}+\alpha_{3}\right] k_{j}^{2}\left(\frac{\dot{J}_{m}\left(\xi_{j} a\right)}{\xi_{j} a}-\frac{m^{2}}{\left(\xi_{j} a\right)^{2}} J_{m}\left(\xi_{j} a\right)\right)+\frac{1}{2} \alpha_{3} k_{j}^{2} J_{m}\left(\xi_{j} a\right) \\
& -2\left[\alpha_{4}+\alpha_{5}\right] \xi_{j}^{2} \ddot{J}_{m}\left(\xi_{j} a\right)+\frac{1}{2}\left[2 \alpha_{4}+3 \alpha_{5}\right] k_{j}^{2} \ddot{J}_{m}\left(\xi_{j} a\right), j=3,4 \\
& \Gamma_{1,7, j}^{m}=\left.\frac{1}{2}\left[\alpha_{1}+\alpha_{3}+2 \alpha_{4}+3 \alpha_{5}\right] k_{j}^{2} \frac{p^{2}}{\xi_{j}^{2}} i m \frac{d}{d\left(\xi_{j} r\right)}\left(\frac{J_{m}\left(\xi_{j} r\right)}{\left(\xi_{j} r\right)}\right)\right|_{r=a} \\
& \Delta_{1,7, j}^{m}=-\frac{1}{2}\left[\alpha_{1}+\alpha_{3}\right] k_{j}^{2}\left(\frac{\dot{J}_{m}\left(\xi_{j} a\right)}{\xi_{j} a}-\frac{m^{2}}{\left(\xi_{j} a\right)^{2}} J_{m}\left(\xi_{j} a\right)\right)+\frac{1}{2} \alpha_{3} k_{j}^{2} J_{m}\left(\xi_{j} a\right) \\
& -2\left[\alpha_{4}+\alpha_{5}\right] \xi_{j}^{2} \ddot{J}_{m}\left(\xi_{j} a\right)+\frac{1}{2}\left[2 \alpha_{4}+3 \alpha_{5}\right] k_{j}^{2} \ddot{J}_{m}\left(\xi_{j} a\right) \\
& E_{1,7, j}^{m}=\left.\frac{1}{2}\left[\alpha_{1}+\alpha_{3}+2 \alpha_{4}+3 \alpha_{5}\right] k_{j}^{2} i m \frac{d}{d\left(\xi_{j} r\right)}\left(\frac{J_{m}\left(\xi_{j} r\right)}{\left(\xi_{j} r\right)}\right)\right|_{r=a} \\
& Z_{1,7, j}^{m}=\frac{1}{2}\left[\alpha_{1}+\alpha_{3}\right] k_{j}^{2} \frac{p^{2}}{\xi_{j}^{2}}\left(\frac{\dot{J}_{m}\left(\xi_{j} a\right)}{\xi_{j} a}-\frac{m^{2}}{\left(\xi_{j} a\right)^{2}} J_{m}\left(\xi_{j} a\right)\right)-\frac{1}{2} \alpha_{3} k_{j}^{2} \frac{p^{2}}{\xi_{j}^{2}} J_{m}\left(\xi_{j} a\right) \\
& +2\left[\alpha_{4}+\alpha_{5}\right] p^{2} \ddot{J}_{m}\left(\xi_{j} a\right)-\frac{1}{2}\left[2 \alpha_{4}+3 \alpha_{5}\right] k_{j}^{2} \frac{p^{2}}{\xi_{j}^{2}} \ddot{J}_{m}\left(\xi_{j} a\right)
\end{aligned}
$$

1.8. Projection on $\left(\hat{\mathbf{r}} \mathbf{C}_{m}\right)_{\alpha}$

$$
A_{1,8, j}^{m}=-\frac{1}{2}\left[3 \alpha_{1}+2 \alpha_{2}+2 \alpha_{3}\right] k_{j}^{2} \frac{p}{\xi_{j}} \dot{J}_{m}\left(\xi_{j} a\right), j=1,2
$$




$$
\begin{gathered}
B_{1,8, j}^{m}=\frac{1}{2}\left[\alpha_{1}+2 \alpha_{3}\right] k_{j}^{2} \frac{p}{\xi_{j}} i m \frac{J_{m}\left(\xi_{j} a\right)}{\left(\xi_{j} a\right)} \\
-\left.2\left[\alpha_{4}+\alpha_{5}\right] p \xi_{j} i m \frac{d^{2}}{d\left(\xi_{j} r\right)^{2}}\left(\frac{J_{m}\left(\xi_{j} r\right)}{\left(\xi_{j} r\right)}\right)\right|_{r=a} \cdot j=1,2 \\
C_{1,8, j}^{m}=-\frac{1}{2}\left[3 \alpha_{1}+2 \alpha_{2}+2 \alpha_{3}\right] k_{j}^{2} \frac{p}{\xi_{j}} \dot{J}_{m}\left(\xi_{j} a\right)-\frac{1}{2}\left[\alpha_{1}+2 \alpha_{3}\right] k_{j}^{2} \frac{p}{\xi_{j}} \frac{k_{j}^{2}}{\xi_{j}^{2}} \dot{J}_{m}\left(\xi_{j} a\right) \\
+2\left[\alpha_{4}+\alpha_{5}\right]\left(p \xi_{j}\right) \frac{k_{j}^{2}}{\xi_{j}^{2}} J_{m}^{(i i i)}\left(\xi_{j} a\right) \\
A_{1,8, j}^{m}=-\frac{1}{2}\left[\alpha_{1}+\alpha_{3}+2 \alpha_{4}+3 \alpha_{5}\right] k_{j}^{2} \frac{p}{\xi_{j}} \dot{J}_{m}\left(\xi_{j} a\right), j=3,4 \\
B_{1,8, j}^{m}=-\frac{1}{2}\left[\alpha_{1}+2 \alpha_{3}\right] k_{j}^{2} \frac{p}{\xi_{j}} i m \frac{J_{m}\left(\xi_{j} a\right)}{\left(\xi_{j} a\right)} \\
+\left.2\left[\alpha_{4}+\alpha_{5}\right] p \xi_{j} i m \frac{d^{2}}{d\left(\xi_{j} r\right)^{2}}\left(\frac{J_{m}\left(\xi_{j} r\right)}{\left(\xi_{j} r\right)}\right)\right|_{r=a}, j=3,4 \\
Z_{1,8, j}^{m}=\frac{1}{2}\left[\alpha_{1}+\alpha_{3}\right] k_{j}^{2} \frac{p^{3}}{\xi_{j}^{3}} \dot{J}_{m}\left(\xi_{j} a\right)+\frac{1}{2} \alpha_{3} k_{j}^{2} \frac{p}{\xi_{j}} \frac{k_{j}^{2}}{\xi_{j}^{2}} \dot{J}_{m}\left(\xi_{j} a\right) \\
-2\left[\alpha_{4}+\alpha_{5}\right]\left(p \xi_{j}\right) \frac{k_{j}^{2}}{\xi_{j}^{2}} J_{m}^{(i i i)}\left(\xi_{j} a\right)-\frac{1}{2}\left[2 \alpha_{4}+3 \alpha_{5}\right] k_{j}^{2} \frac{p}{\xi_{j}} \dot{J}_{m}\left(\xi_{j} a\right) \\
+\left.2\left[\alpha_{4}+\alpha_{5}\right] p \xi_{j} i m \frac{d^{2}}{d\left(\xi_{j} r\right)^{2}}\left(\frac{J_{m}\left(\xi_{j} r\right)}{\left(\xi_{j} r\right)}\right)\right|_{r=a} \\
\Delta_{1,8, j}^{m}=-\frac{1}{2}\left[\alpha_{1}+2 \alpha_{3}\right] k_{j}^{2} \frac{p}{\xi_{j}} i m \frac{J_{m}\left(\xi_{j} a\right)}{\left(\xi_{j} a\right)} \\
+\left.2\left[\alpha_{4}+\alpha_{5}\right] p \xi_{j} i m \frac{d^{2}}{d\left(\xi_{j} r\right)^{2}}\left(\frac{J_{m}\left(\xi_{j} r\right)}{\left(\xi_{j} r\right)}\right)\right|_{r=a}
\end{gathered}
$$

1.9. Projection on $\left(\hat{\phi} \mathbf{C}_{m}\right)_{\alpha}$

$$
\begin{gathered}
A_{1,9, j}^{m}=0, j=1,2 \\
B_{1,9, j}^{m}=-\frac{1}{2}\left[\alpha_{1}+2 \alpha_{3}\right] k_{j}^{2} \frac{p}{\xi_{j}} \dot{J}_{m}\left(\xi_{j} a\right)+2\left[\alpha_{4}+\alpha_{5}\right] p \xi_{j} J_{m}^{(i i i)}\left(\xi_{j} a\right), j=1,2(\text { A.70) }
\end{gathered}
$$




$$
\begin{gathered}
C_{1,9, j}^{m}=-\frac{1}{2}\left[\alpha_{1}+2 \alpha_{3}\right] k_{j}^{2} \frac{p}{\xi_{j}} \frac{k_{j}^{2}}{\xi_{j}^{2}} i m \frac{J_{m}\left(\xi_{j} a\right)}{\left(\xi_{j} a\right)} \\
+\left.2\left[\alpha_{4}+\alpha_{5}\right] p \xi_{j} \frac{k_{j}^{2}}{\xi_{j}^{2}} i m \frac{d^{2}}{d\left(\xi_{j} r\right)^{2}}\left(\frac{J_{m}\left(\xi_{j} r\right)}{\left(\xi_{j} r\right)}\right)\right|_{r=a} \\
A_{1,9, j}^{m}=0, j=3,4 \\
B_{1,9, j}^{m}=\frac{1}{2} \alpha_{3} k_{j}^{2} \frac{p}{\xi_{j}} \dot{J}_{m}\left(\xi_{j} a\right)-2\left[\alpha_{4}+\alpha_{5}\right] p \xi_{j} J_{m}^{(i i i)}\left(\xi_{j} a\right), j=3,4 \\
\Gamma_{1,9, j}^{m}=\frac{1}{2} \alpha_{3} k_{j}^{2} \frac{p}{\xi_{j}} \frac{k_{j}^{2}}{\xi_{j}^{2}} i m \frac{J_{m}\left(\xi_{j} a\right)}{\left(\xi_{j} a\right)} \\
-\left.2\left[\alpha_{4}+\alpha_{5}\right] p \xi_{j} \frac{k_{j}^{2}}{\xi_{j}^{2}} i m \frac{d^{2}}{d\left(\xi_{j} r\right)^{2}}\left(\frac{J_{m}\left(\xi_{j} r\right)}{\left(\xi_{j} r\right)}\right)\right|_{r=a} \\
\Delta_{1,9, j}^{m}=\Delta_{1,5, j}^{m}, \quad E_{1,9, j}^{m}=0, \quad Z_{1,9, j}^{m}=\Delta_{1,9, j}^{m}
\end{gathered}
$$

2. The coefficients offered by boundary condition (41).

We introduce first some helpful entities which appear repeatedly in the sequel. 2.1. The following auxiliary quantities are necessary:

$$
\begin{aligned}
& \Lambda_{j}=\frac{1}{2}\left[\alpha_{1}+3 \alpha_{3}+2 \alpha_{4}+\alpha_{5}\right] k_{j}^{2}-2\left[\alpha_{4}+\alpha_{5}\right] p^{2} \\
& \Lambda_{j}^{\prime}=\Lambda_{j}+\frac{1}{2}\left[5 \alpha_{1}+4 \alpha_{2}+4 \alpha_{3}+2 \alpha_{4}+3 \alpha_{5}\right] k_{j}^{2} \\
& X_{j}=2 \mu+\frac{1}{2}\left[\alpha_{1}+3 \alpha_{3}+6 \alpha_{4}+5 \alpha_{5}\right] k_{j}^{2}-\rho \omega^{2}\left(2 h_{2}^{2}-\frac{\rho^{\prime} d^{2}}{3 \rho}\right) \\
& X_{j}^{\prime}=X_{j}+\frac{1}{2}\left[4 \alpha_{1}+4 \alpha_{2}+3 \alpha_{3}+2 \alpha_{4}+3 \alpha_{5}\right] k_{j}^{2} \\
& T_{j}=\mu k_{j}^{2}+\frac{1}{2}\left[\alpha_{1}+\alpha_{3}+2 \alpha_{4}+3 \alpha_{5}\right] k_{j}^{4}-k_{j}^{2} \rho \omega^{2}\left(h_{2}^{2}-\frac{\rho^{\prime} d^{2}}{3 \rho}\right) \\
& Q_{j}=-\lambda k_{j}^{2}-\frac{1}{2}\left[3 \alpha_{1}+4 \alpha_{2}+2 \alpha_{3}\right] k_{j}^{4}+k_{j}^{2} \rho \omega^{2}\left(h_{1}^{2}-2 h_{2}^{2}+\frac{\rho^{\prime} d^{2}}{3 \rho}\right)
\end{aligned}
$$

2.2. Let us introduce the "inner products" $U_{l, q, j}^{m}$, induced by projecting the traces (on the cylinder surface $r=a$ ) of the dyadic eigensolutions on the dyadic harmonics. The first index $l$ refers to eigensolution numbering while the second 
one $q$ pertains to the dyadic harmonic enumeration. We then obtain

$$
\begin{aligned}
& U_{1,1, j}^{m}=\ddot{J}_{m}\left(\xi_{j} a\right), U_{2,1, j}^{m}=\left.i m \frac{d}{d\left(\xi_{j} r\right)}\left(\frac{J_{m}\left(\xi_{j} r\right)}{\left(\xi_{j} r\right)}\right)\right|_{r=a}, j=1,2(\mathrm{~A} .76) \\
& U_{3,1, j}^{m}=-\frac{p^{2}}{\xi_{j}^{2}} \ddot{J}_{m}\left(\xi_{j} a\right), j=1,2
\end{aligned}
$$

(note again that, for example, $U_{1,1, j}^{m}(r)=\ddot{J}_{m}\left(\xi_{j} r\right)$ )

$$
\begin{aligned}
& U_{4,1, j}^{m}=\ddot{J}_{m}\left(\xi_{j} a\right)+\frac{k_{j}^{2}}{\xi_{j}^{2}} \ddot{J}_{m}\left(\xi_{j} a\right), U_{5,1, j}^{m}=\left.i m \frac{d}{d\left(\xi_{j} r\right)}\left(\frac{J_{m}\left(\xi_{j} r\right)}{\left(\xi_{j} r\right)}\right)\right|_{r=a}, \\
& U_{6,1, j}^{m}=-\frac{p^{2}}{\xi_{j}^{2}} \ddot{J}_{m}\left(\xi_{j} a\right), U_{7,1, j}^{m}=U_{5,1, j}^{m} \\
& U_{8,1, j}^{m}=\frac{\dot{J}_{m}\left(\xi_{j} a\right)}{\xi_{j} a}-\frac{m^{2}}{\left(\xi_{j} a\right)^{2}} J_{m}\left(\xi_{j} a\right), \\
& U_{9,1, j}^{m}=-\left.i m \frac{p^{2}}{\xi_{j}^{2}} \frac{d}{d\left(\xi_{j} r\right)}\left(\frac{J_{m}\left(\xi_{j} r\right)}{\left(\xi_{j} r\right)}\right)\right|_{r=a}, j=3,4 \\
& U_{1,2, j}^{m}=2 U_{2,1, j}^{m}, \quad U_{2,2, j}^{m}=2\left(\frac{\dot{J}_{m}\left(\xi_{j} a\right)}{\xi_{j} a}-\frac{m^{2}}{\left(\xi_{j} a\right)^{2}} J_{m}\left(\xi_{j} a\right)\right)+J_{m}\left(\xi_{j} a\right), \\
& U_{3,2, j}^{m}=-\left.2 \frac{p^{2}}{\xi_{j}^{2}} i m \frac{d}{d\left(\xi_{j} r\right)}\left(\frac{J_{m}\left(\xi_{j} r\right)}{\left(\xi_{j} r\right)}\right)\right|_{r=a}, j=1,2 \\
& U_{4,2, j}^{m}=2 U_{5,1, j}^{m}, U_{5,2, j}^{m}=2\left(\frac{\dot{J}_{m}\left(\xi_{j} a\right)}{\xi_{j} a}-\frac{m^{2}}{\left(\xi_{j} a\right)^{2}} J_{m}\left(\xi_{j} a\right)\right)+J_{m}\left(\xi_{j} a\right) \\
& U_{6,2, j}^{m}=-\left.2 \frac{p^{2}}{\xi_{j}^{2}} i m \frac{d}{d\left(\xi_{j} r\right)}\left(\frac{J_{m}\left(\xi_{j} r\right)}{\left(\xi_{j} r\right)}\right)\right|_{r=a}, U_{7,2, j}^{m}=U_{5,2, j}^{m} \\
& U_{8,2, j}^{m}=-U_{4,2, j}^{m}, U_{9,2, j}^{m}=-\frac{p^{2}}{\xi_{j}^{2}} U_{5,2, j}^{m}, j=3,4 \\
& U_{1,3, j}^{m}=\frac{\dot{J}_{m}\left(\xi_{j} a\right)}{\xi_{j} a}-\frac{m^{2}}{\left(\xi_{j} a\right)^{2}} J_{m}\left(\xi_{j} a\right), U_{2,3, j}^{m}=-\left.i m \frac{d}{d\left(\xi_{j} r\right)}\left(\frac{J_{m}\left(\xi_{j} r\right)}{\left(\xi_{j} r\right)}\right)\right|_{r=a}, \\
& U_{3,3, j}^{m}=-\frac{p^{2}}{\xi_{j}^{2}}\left(\frac{\dot{J}_{m}\left(\xi_{j} a\right)}{\xi_{j} a}-\frac{m^{2}}{\left(\xi_{j} a\right)^{2}} J_{m}\left(\xi_{j} a\right)\right), j=1,2
\end{aligned}
$$




$$
\begin{aligned}
& U_{4,3, j}^{m}=\frac{\dot{J}_{m}\left(\xi_{j} a\right)}{\xi_{j} a}+\left(\frac{k_{j}^{2}}{\xi_{j}^{2}}-\frac{m^{2}}{\left(\xi_{j} a\right)^{2}}\right) J_{m}\left(\xi_{j} a\right), \\
& U_{5,3, j}^{m}=-\left.i m \frac{d}{d\left(\xi_{j} r\right)}\left(\frac{J_{m}\left(\xi_{j} r\right)}{\left(\xi_{j} r\right)}\right)\right|_{r=a} \\
& U_{6,3, j}^{m}=-\frac{p^{2}}{\xi_{j}^{2}}\left(\frac{\dot{J}_{m}\left(\xi_{j} a\right)}{\xi_{j} a}-\frac{m^{2}}{\left(\xi_{j} a\right)^{2}} J_{m}\left(\xi_{j} a\right)\right) \text {, } \\
& U_{7,3, j}^{m}=U_{5,3, j}^{m}, U_{8,3, j}^{m}=\ddot{J}_{m}\left(\xi_{j} a\right), U_{9,3, j}^{m}=-\frac{p^{2}}{\xi_{j}^{2}} U_{5,3, j}^{m}, j=3,4(\mathrm{~A} .82) \\
& U_{1,4, j}^{m}=2 \frac{p}{\xi_{j}} \dot{J}_{m}\left(\xi_{j} a\right), \\
& U_{2,4, j}^{m}=i m \frac{p}{\xi_{j}} \frac{J_{m}\left(\xi_{j} a\right)}{\left(\xi_{j} a\right)}, U_{3,4, j}^{m}=\frac{1}{2}\left(1-\frac{p^{2}}{\xi_{j}^{2}}\right) U_{1,4, j}^{m}, j=1,2 \\
& U_{4,4, j}^{m}=2 \frac{p}{\xi_{j}} \dot{J}_{m}\left(\xi_{j} a\right), U_{5,4, j}^{m}=i m \frac{p}{\xi_{j}} \frac{J_{m}\left(\xi_{j} a\right)}{\left(\xi_{j} a\right)}, U_{6,4, j}^{m}=\frac{1}{2}\left(1-\frac{p^{2}}{\xi_{j}^{2}}\right) U_{4,4, j}^{m}, \\
& U_{7,4, j}^{m}=U_{5,4, j}^{m}, \quad U_{8,4, j}^{m}=0, U_{9,4, j}^{m}=U_{5,4, j}^{m}, j=3,4 \\
& U_{1,5, j}^{m}=2 i m \frac{p}{\xi_{j}} \frac{J_{m}\left(\xi_{j} a\right)}{\left(\xi_{j} a\right)} \\
& U_{2,5, j}^{m}=-\frac{p}{\xi_{j}} \dot{J}_{m}\left(\xi_{j} a\right), U_{3,5, j}^{m}=\frac{1}{2}\left(1-\frac{p^{2}}{\xi_{j}^{2}}\right) U_{1,5, j}^{m}, j=1,2 \\
& U_{4,5, j}^{m}=2 i m \frac{p}{\xi_{j}} \frac{J_{m}\left(\xi_{j} a\right)}{\left(\xi_{j} a\right)}, U_{5,5, j}^{m}=-\frac{p}{\xi_{j}} \dot{J}_{m}\left(\xi_{j} a\right), U_{6,5, j}^{m}=\frac{1}{2}\left(1-\frac{p^{2}}{\xi_{j}^{2}}\right) U_{4,5, j}^{m}, \\
& U_{7,5, j}^{m}=U_{5,5, j}^{m}, U_{8,5, j}^{m}=0, U_{9,5, j}^{m}=U_{5,5, j}^{m}, j=3,4 \\
& U_{1,6, j}^{m}=-\frac{p^{2}}{\xi_{j}^{2}} J_{m}\left(\xi_{j} a\right), U_{2,6, j}^{m}=0, U_{3,6, j}^{m}=U_{1,6, j}^{m}, j=1,2 \\
& U_{4,6, j}^{m}=J_{m}\left(\xi_{j} a\right), U_{5,6, j}^{m}=0, U_{6,6, j}^{m}=-\frac{p^{2}}{\xi_{j}^{2}} J_{m}\left(\xi_{j} a\right), \\
& U_{7,6, j}^{m}=U_{8,6, j}^{m}=U_{9,6, j}^{m}=0, j=3,4 \\
& U_{1,7, j}^{m}=0, U_{2,7, j}^{m}=J_{m}\left(\xi_{j} a\right), U_{3,7, j}^{m}=0, j=1,2 \\
& U_{4,7, j}^{m}=0, U_{5,7, j}^{m}=-J_{m}\left(\xi_{j} a\right), U_{6,7, j}^{m}=0, U_{7,7, j}^{m}=U_{5,7, j}^{m}, \\
& U_{8,7, j}^{m}=0, U_{9,7, j}^{m}=\frac{p^{2}}{\xi_{j}^{2}} J_{m}\left(\xi_{j} a\right), j=3,4
\end{aligned}
$$




$$
\begin{gathered}
U_{1,8, j}^{m}=0, U_{2,8, j}^{m}=-i m \frac{p}{\xi_{j}} \frac{J_{m}\left(\xi_{j} a\right)}{\left(\xi_{j} a\right)}, \\
U_{3,8, j}^{m}=\frac{p}{\xi_{j}} \frac{k_{j}^{2}}{\xi_{j}^{2}} \dot{J}_{m}\left(\xi_{j} a\right), j=1,2 \\
U_{4,8, j}^{m}=0, U_{5,8, j}^{m}=i m \frac{p}{\xi_{j}} \frac{J_{m}\left(\xi_{j} a\right)}{\left(\xi_{j} a\right)}, U_{6,8, j}^{m}=-\frac{p}{\xi_{j}} \frac{k_{j}^{2}}{\xi_{j}^{2}} \dot{J}_{m}\left(\xi_{j} a\right), \\
U_{7,8, j}^{m}=U_{5,8, j}^{m}, U_{8,8, j}^{m}=0, U_{9,8, j}^{m}=U_{5,8, j}^{m}, j=3,4 \\
U_{1,9, j}^{m}=0, U_{2,9, j}^{m}=\frac{p}{\xi_{j}} \dot{J}_{m}\left(\xi_{j} a\right), \\
U_{3,9, j}^{m}=i m \frac{p}{\xi_{j}} \frac{k_{j}^{2}}{\xi_{j}^{2}} \frac{J_{m}\left(\xi_{j} a\right)}{\left(\xi_{j} a\right)}, j=1,2 \\
U_{4,9, j}^{m}=0, U_{5,9, j}^{m}=-\frac{p}{\xi_{j}} \dot{J}_{m}\left(\xi_{j} a\right), U_{6,9, j}^{m}=-i m \frac{p}{\xi_{j}} \frac{k_{j}^{2}}{\xi_{j}^{2}} \frac{J_{m}\left(\xi_{j} a\right)}{\left(\xi_{j} a\right)} \\
U_{7,9, j}^{m}=U_{5,9, j}^{m}, U_{8,9, j}^{m}=0, U_{9,9, j}^{m}=U_{5,9, j}^{m}, j=3,4
\end{gathered}
$$

2.3. We have at hand all the necessary quantities to form the coefficients appearing in Eq.(43) (with $s=2$ ):

2.3.1. Projection on $\hat{\mathbf{r}} \mathbf{P}_{m}$

$$
\begin{gathered}
A_{2,1, j}^{m}=\frac{\Lambda_{j}^{\prime}}{a} U_{1,1, j}^{m}+X_{j}^{\prime} U_{1,1, j}^{\prime m}+\frac{A_{1,1, j}^{m}}{a}+2 A_{1,1, j}^{\prime m}+Q_{j} \frac{\dot{J}_{m}\left(\xi_{j} a\right)}{\xi_{j}}, j=1,2(\mathrm{~A} .95) \\
\left.B_{2,1, j}^{m}=\frac{\Lambda_{j}^{\prime}}{a} U_{2,1, j}^{m}+X_{j}^{\prime} U_{2,1, j}^{\prime m}+\frac{B_{1,1, j}^{m}}{a}+2 B_{1,1, j}^{\prime m}+Q_{j} i m \frac{J_{m}\left(\xi_{j} a\right)}{\xi_{j}^{2} a}, j=1,2 \mathrm{~A} .96\right) \\
C_{2,1, j}^{m}=\frac{\Lambda_{j}^{\prime}}{a} U_{3,1, j}^{m}+X_{j}^{\prime} U_{3,1, j}^{\prime m}+\frac{C_{1,1, j}^{m}}{a}+2 C_{1,1, j}^{\prime m}-Q_{j} \frac{p^{2}}{\xi_{j}^{2}} \frac{\dot{J}_{m}\left(\xi_{j} a\right)}{\xi_{j}} \quad \text { (A.97) } \\
A_{2,1, j}^{m}=\frac{\Lambda_{j}}{a} U_{4,1, j}^{m}+X_{j} U_{4,1, j}^{\prime m}+\frac{A_{1,1, j}^{m}}{a}+2 A_{1,1, j}^{\prime m} \\
+\frac{1}{2}\left[2 \alpha_{4}+3 \alpha_{5}\right] k_{j}^{2} \frac{p^{2}}{\xi_{j}^{2}} \frac{J_{m}\left(\xi_{j} a\right)}{a}, j=3,4 \\
B_{2,1, j}^{m}=\frac{\Lambda_{j}}{a} U_{5,1, j}^{m}+X_{j} U_{5,1, j}^{\prime m}+\frac{B_{1,1, j}^{m}}{a}+2 B_{1,1, j}^{\prime m}, j=3,4
\end{gathered}
$$




$$
\begin{gathered}
\Gamma_{2,1, j}^{m}=\frac{\Lambda_{j}}{a} U_{6,1, j}^{m}+X_{j} U_{6,1, j}^{\prime m}+\frac{\Gamma_{1,1, j}^{m}}{a}+2 \Gamma_{1,1, j}^{\prime m} \\
+\frac{1}{2}\left[2 \alpha_{4}+3 \alpha_{5}\right] k_{j}^{2} \frac{p^{2}}{\xi_{j}^{2}} \frac{J_{m}\left(\xi_{j} a\right)}{a} \\
\Delta_{2,1, j}^{m}=\frac{\Lambda_{j}}{a} U_{7,1, j}^{m}+X_{j} U_{7,1, j}^{\prime m}+\frac{\Delta_{1,1, j}^{m}}{a}+2 \Delta_{1,1, j}^{\prime m} \\
E_{2,1, j}^{m}=\frac{\Lambda_{j}}{a} U_{8,1, j}^{m}+X_{j} U_{8,1, j}^{\prime m}+\frac{E_{1,1, j}^{m}}{a}+2 E_{1,1, j}^{\prime m} \\
Z_{2,1, j}^{m}=\frac{\Lambda_{j}}{a} U_{9,1, j}^{m}+X_{j} U_{9,1, j}^{\prime m}+\frac{Z_{1,1, j}^{m}}{a}+2 Z_{1,1, j}^{\prime m}
\end{gathered}
$$

2.3.2. Projection on $\left(\hat{\mathbf{r}} \mathbf{B}_{m}\right)_{s}$

$$
\begin{aligned}
& A_{2,2, j}^{m}=\frac{\Lambda_{j}^{\prime}}{a} U_{1,2, j}^{m}+X_{j}^{\prime} U_{1,2, j}^{\prime m}+\frac{A_{1,2, j}^{m}}{a}+2 A_{1,2, j}^{\prime m}+Q_{j} i m \frac{J_{m}\left(\xi_{j} a\right)}{\xi_{j}^{2} a}, j=1(\mathbf{\AA} .104) \\
& B_{2,2, j}^{m}=\frac{\Lambda_{j}^{\prime}}{a} U_{2,2, j}^{m}+X_{j}^{\prime} U_{2,2, j}^{\prime m}+\frac{B_{1,2, j}^{m}}{a}+2 B_{1,2, j}^{\prime m}-Q_{j} \frac{\dot{J}_{m}\left(\xi_{j} a\right)}{\xi_{j}}, j=1(\text { \.105) } \\
& C_{2,2, j}^{m}=\frac{\Lambda_{j}^{\prime}}{a} U_{3,2, j}^{m}+X_{j}^{\prime} U_{3,2, j}^{\prime m}+\frac{C_{1,2, j}^{m}}{a}+2 C_{1,2, j}^{\prime m}-Q_{j} i m \frac{p^{2}}{\xi_{j}^{3}} \frac{J_{m}\left(\xi_{j} a\right)}{\xi_{j} a}(\mathrm{~A} .106) \\
& A_{2,2, j}^{m}=\frac{\Lambda_{j}}{a} U_{4,2, j}^{m}+X_{j} U_{4,2, j}^{\prime m}+\frac{A_{1,2, j}^{m}}{a}+2 A_{1,2, j}^{\prime m}+T_{j} i m \frac{J_{m}\left(\xi_{j} a\right)}{\xi_{j}^{2} a}, j=3,(\mathbb{A} .107) \\
& B_{2,2, j}^{m}=\frac{\Lambda_{j}}{a} U_{5,2, j}^{m}+X_{j} U_{5,2, j}^{\prime m}+\frac{B_{1,2, j}^{m}}{a}+2 B_{1,2, j}^{\prime m}-T_{j} \frac{\dot{J}_{m}\left(\xi_{j} a\right)}{\xi_{j}}, j=3,4 \text { (A.108) } \\
& \Gamma_{2,2, j}^{m}=\frac{\Lambda_{j}}{a} U_{6,2, j}^{m}+X_{j} U_{6,2, j}^{\prime m}+\frac{\Gamma_{1,2, j}^{m}}{a}+2 \Gamma_{1,2, j}^{\prime m}-T_{j} \frac{p^{2}}{\xi_{j}^{3}} i m \frac{J_{m}\left(\xi_{j} a\right)}{\xi_{j} a} \\
& \Delta_{2,2, j}^{m}=\frac{\Lambda_{j}}{a} U_{7,2, j}^{m}+X_{j} U_{7,2, j}^{\prime m}+\frac{\Delta_{1,2, j}^{m}}{a}+2 \Delta_{1,2, j}^{\prime m}-T_{j} \frac{\dot{J}_{m}\left(\xi_{j} a\right)}{\xi_{j}} \\
& E_{2,2, j}^{m}=\frac{\Lambda_{j}}{a} U_{8,2, j}^{m}+X_{j} U_{8,2, j}^{\prime m}+\frac{E_{1,2, j}^{m}}{a}+2 E_{1,2, j}^{\prime m}-T_{j} i m \frac{J_{m}\left(\xi_{j} a\right)}{\xi_{j}^{2} a}
\end{aligned}
$$




$$
Z_{2,2, j}^{m}=\frac{\Lambda_{j}}{a} U_{9,2, j}^{m}+X_{j} U_{9,2, j}^{\prime m}+\frac{Z_{1,2, j}^{m}}{a}+2 Z_{1,2, j}^{\prime m}+T_{j} \frac{p^{2}}{\xi_{j}^{2}} \frac{\dot{j}_{m}\left(\xi_{j} a\right)}{\xi_{j}}
$$

2.3.3. Projection on $\hat{\phi} \mathbf{B}_{m}$

$$
\begin{gathered}
A_{2,3, j}^{m}=\frac{\Lambda_{j}^{\prime}}{a} U_{1,3, j}^{m}+X_{j}^{\prime} U_{1,3, j}^{\prime m}+\frac{A_{1,3, j}^{m}}{a}+2 A_{1,3, j}^{\prime m}, j=1,2 \\
B_{2,3, j}^{m}=\frac{\Lambda_{j}^{\prime}}{a} U_{2,3, j}^{m}+X_{j}^{\prime} U_{2,3, j}^{\prime m}+\frac{B_{1,3, j}^{m}}{a}+2 B_{1,3, j}^{\prime m}, j=1,2 \\
C_{2,3, j}^{m}=\frac{\Lambda_{j}^{\prime}}{a} U_{3,3, j}^{m}+X_{j}^{\prime} U_{3,3, j}^{\prime m}+\frac{C_{1,3, j}^{m}}{a}+2 C_{1,3, j}^{\prime m} \\
A_{2,3, j}^{m}=\frac{\Lambda_{j}}{a} U_{4,3, j}^{m}+X_{j} U_{4,3, j}^{\prime m}+\frac{A_{1,3, j}^{m}}{a}+2 A_{1,3, j}^{\prime m} \\
-T_{j} \frac{\dot{J}_{m}\left(\xi_{j} a\right)}{\xi_{j}}+\frac{1}{2}\left[2 \alpha_{4}+3 \alpha_{5}\right] k_{j}^{2} \frac{p^{2}}{\xi_{j}^{2}} \frac{J_{m}\left(\xi_{j} a\right)}{a}, j=3,4 \\
B_{2,3, j}^{m}=\frac{\Lambda_{j}}{a} U_{5,3, j}^{m}+X_{j} U_{5,3, j}^{\prime m}+\frac{B_{1,3, j}^{m}}{a}+2 B_{1,3, j}^{\prime m}-T_{j} i m \frac{J_{m}\left(\xi_{j} a\right)}{\xi_{j}^{2} a}, j=3,(A), 1 \\
\Gamma_{2,3, j}^{m}=\frac{\Lambda_{j}}{a} U_{6,3, j}^{m}+X_{j} U_{6,3, j}^{\prime m}+\frac{\Gamma_{1,3, j}^{m}}{a}+2 \Gamma_{1,3, j}^{\prime m} \\
+T_{j} \frac{p^{2}}{\xi_{j}^{2}} \frac{\dot{J}_{m}\left(\xi_{j} a\right)}{\xi_{j}}+\frac{1}{2}\left[2 \alpha_{4}+3 \alpha_{5}\right] k_{j}^{2} \frac{p^{2}}{\xi_{j}^{2}} \frac{J_{m}\left(\xi_{j} a\right)}{a} \\
Z_{2,3, j}^{m}=\frac{\Lambda_{j}}{a} U_{9,3, j}^{m}+X_{j} U_{9,3, j}^{\prime m}+\frac{Z_{1,3, j}^{m}}{a}+2 Z_{1,3, j}^{\prime m}+T_{j} i m \frac{p^{2}}{\xi_{j}^{2}} \frac{J_{m}\left(\xi_{j} a\right)}{\xi_{j}^{2} a} \\
\Delta_{2,3, j}^{m}=\frac{\Lambda_{j}}{a} U_{7,3, j}^{m}+X_{j} U_{7,3, j}^{\prime m}+\frac{\Delta_{1,3, j}^{m}}{a}+2 \Delta_{1,3, j}^{\prime m}-T_{j} i m \frac{J_{m}\left(\xi_{j} a\right)}{\xi_{j}^{2} a} \\
E_{2,3, j}^{m}=\frac{\Lambda_{j}}{a} U_{8,3, j}^{m}+X_{j} U_{8,3, j}^{\prime m}+\frac{E_{1,3, j}^{m}}{a}+2 E_{1,3, j}^{\prime m}+T_{j} \frac{\cdot J_{m}\left(\xi_{j} a\right)}{\xi_{j}}
\end{gathered}
$$

2.3.4.Projection on $\left(\hat{\mathbf{r}} \mathbf{C}_{m}\right)_{s}$

$$
\begin{aligned}
& A_{2,4, j}^{m}=\frac{\Lambda_{j}^{\prime}}{a} U_{1,4, j}^{m}+X_{j}^{\prime} U_{1,4, j}^{\prime m}+\frac{A_{1,4, j}^{m}}{a}+2 A_{1,4, j}^{\prime m} \\
& +Q_{j} \frac{p}{\xi_{j}} \frac{J_{m}\left(\xi_{j} a\right)}{\xi_{j}}-\frac{1}{2}\left[4 \alpha_{1}+2 \alpha_{2}+3 \alpha_{3}\right] k_{j}^{2} \frac{p}{\xi_{j}} \frac{\dot{J}_{m}\left(\xi_{j} a\right)}{a}, j=1,2(\mathrm{~A} .122)
\end{aligned}
$$




$$
\begin{gathered}
B_{2,4, j}^{m}=\frac{\Lambda_{j}^{\prime}}{a} U_{2,4, j}^{m}+X_{j}^{\prime} U_{2,4, j}^{\prime m}+\frac{B_{1,4, j}^{m}}{a}+2 B_{1,4, j}^{\prime m} \\
-\frac{1}{2}\left[4 \alpha_{1}+2 \alpha_{2}+3 \alpha_{3}\right] k_{j}^{2} i m \frac{p}{\xi_{j}} \frac{J_{m}\left(\xi_{j} a\right)}{\xi_{j} a^{2}}, j=1,2 \\
C_{2,4, j}^{m}=\frac{\Lambda_{j}^{\prime}}{a} U_{3,4, j}^{m}+X_{j}^{\prime} U_{3,4, j}^{\prime m}+\frac{C_{1,4, j}^{m}}{a}+2 C_{1,4, j}^{\prime m} \\
+Q_{j} \frac{p}{\xi_{j}} \frac{J_{m}\left(\xi_{j} a\right)}{\xi_{j}}+\frac{1}{2}\left[4 \alpha_{1}+2 \alpha_{2}+3 \alpha_{3}\right] k_{j}^{2} \frac{p^{3}}{\xi_{j}^{3}} \frac{\dot{J}_{m}\left(\xi_{j} a\right)}{a} \\
A_{2,4, j}^{m}=\frac{\Lambda_{j}}{a} U_{4,4, j}^{m}+X_{j} U_{4,4, j}^{\prime m}+\frac{A_{1,4, j}^{m}}{a}+2 A_{1,4, j}^{\prime m}+T_{j} \frac{p}{\xi_{j}} \frac{J_{m}\left(\xi_{j} a\right)}{\xi_{j}} \\
+\frac{1}{2}\left[2 \alpha_{4}+3 \alpha_{5}-\alpha_{1}-\alpha_{3}\right] k_{j}^{2} \frac{p}{\xi_{j}} \frac{\dot{J}_{m}\left(\xi_{j} a\right)}{a}, j=3,4 \\
Z_{2,4, j}^{m}=\frac{\Lambda_{j}}{a} U_{9,4, j}^{m}+X_{j} U_{9,4, j}^{\prime}+\frac{Z_{1,4, j}^{m}}{a}+2 Z_{1,4, j}^{\prime} \\
E_{2,4, j}^{m}=\frac{1}{2}\left[\alpha_{1}^{m}+\alpha_{3}\right] k_{j}^{2} \frac{p}{\xi_{j}} \frac{\dot{J}_{m}\left(\xi_{j} a\right)}{a}-\frac{1}{2}\left[2 \alpha_{4}+3 \alpha_{5}\right] k_{j}^{2} \frac{p^{3}}{\xi_{j}^{3}} \frac{\dot{J}_{m}\left(\xi_{j} a\right)}{a} \\
B_{2,4, j}^{m}=\frac{\Lambda_{j}}{a} U_{7,4, j}^{m} U_{5,4, j}^{m}+X_{j} U_{5,4, j}^{\prime m}+\frac{B_{1,4, j}^{m}}{a}+2 B_{1,4, j}^{\prime m} \\
+\frac{1}{2}\left[2 \alpha_{4}+3 \alpha_{5}\right] k_{j}^{2} i m \frac{p}{\xi_{j}} \frac{J_{m}\left(\xi_{j} a\right)}{\xi_{j} a^{2}}, j=3,4 \\
\sum_{j}
\end{gathered}
$$

2.3.5. Projection on $\left(\hat{\phi} \mathbf{C}_{m}\right)_{s}$

$$
\begin{aligned}
& A_{2,5, j}^{m}=\frac{\Lambda_{j}^{\prime}}{a} U_{1,5, j}^{m}+X_{j}^{\prime} U_{1,5, j}^{\prime m}+\frac{A_{1,5, j}^{m}}{a}+2 A_{1,5, j}^{\prime m} \\
& -\frac{1}{2}\left[4 \alpha_{1}+2 \alpha_{2}+3 \alpha_{3}\right] k_{j}^{2} i m \frac{p}{\xi_{j}} \frac{J_{m}\left(\xi_{j} a\right)}{\xi_{j} a^{2}}, j=1,2
\end{aligned}
$$




$$
\begin{aligned}
& B_{2,5, j}^{m}=\frac{\Lambda_{j}^{\prime}}{a} U_{2,5, j}^{m}+X_{j}^{\prime} U_{2,5, j}^{\prime m}+\frac{B_{1,5, j}^{m}}{a}+2 B_{1,5, j}^{\prime m} \\
& +\frac{1}{2}\left[4 \alpha_{1}+2 \alpha_{2}+3 \alpha_{3}\right] k_{j}^{2} \frac{p}{\xi_{j}} \frac{\dot{J}_{m}\left(\xi_{j} a\right)}{a}, j=1,2 \\
& C_{2,5, j}^{m}=\frac{\Lambda_{j}^{\prime}}{a} U_{3,5, j}^{m}+X_{j}^{\prime} U_{3,5, j}^{\prime m}+\frac{C_{1,5, j}^{m}}{a}+2 C_{1,5, j}^{\prime m} \\
& +\frac{1}{2}\left[4 \alpha_{1}+2 \alpha_{2}+3 \alpha_{3}\right] k_{j}^{2} i m \frac{p^{3}}{\xi_{j}^{3}} \frac{J_{m}\left(\xi_{j} a\right)}{\xi_{j} a^{2}} \\
& A_{2,5, j}^{m}=\frac{\Lambda_{j}}{a} U_{4,5, j}^{m}+X_{j} U_{4,5, j}^{\prime m}+\frac{A_{1,5, j}^{m}}{a}+2 A_{1,5, j}^{\prime m} \\
& +\frac{1}{2}\left[2 \alpha_{4}+3 \alpha_{5}-\alpha_{1}-\alpha_{3}\right] k_{j}^{2} i m \frac{p}{\xi_{j}} \frac{J_{m}\left(\xi_{j} a\right)}{\xi_{j} a^{2}}, j=3,4 \\
& B_{2,5, j}^{m}=\frac{\Lambda_{j}}{a} U_{5,5, j}^{m}+X_{j} U_{5,5, j}^{\prime m}+\frac{B_{1,5, j}^{m}}{a}+2 B_{1,5, j}^{\prime m} \\
& -\frac{1}{2}\left[2 \alpha_{4}+3 \alpha_{5}\right] k_{j}^{2} \frac{p}{\xi_{j}} \frac{\dot{J}_{m}\left(\xi_{j} a\right)}{a}, j=3,4 \\
& \Gamma_{2,5, j}^{m}=\frac{\Lambda_{j}}{a} U_{6,5, j}^{m}+X_{j} U_{6,5, j}^{\prime m}+\frac{\Gamma_{1,5, j}^{m}}{a}+2 \Gamma_{1,5, j}^{\prime m} \\
& -\frac{1}{2}\left[\alpha_{1}+\alpha_{3}\right] k_{j}^{2} \frac{p}{\xi_{j}} i m \frac{J_{m}\left(\xi_{j} a\right)}{\xi_{j} a^{2}}-\frac{1}{2}\left[2 \alpha_{4}+3 \alpha_{5}\right] k_{j}^{2} \frac{p^{3}}{\xi_{j}^{3}} i m \frac{J_{m}\left(\xi_{j} a\right)}{\xi_{j} a^{2}}(\mathrm{~A} \\
& \Delta_{2,5, j}^{m}=\frac{\Lambda_{j}}{a} U_{7,5, j}^{m}+X_{j} U_{7,5, j}^{\prime m}+\frac{\Delta_{1,5, j}^{m}}{a}+2 \Delta_{1,5, j}^{\prime m}-T_{j} \frac{p}{\xi_{j}} \frac{J_{m}\left(\xi_{j} a\right)}{\xi_{j}} \\
& E_{2,5, j}^{m}=\frac{\Lambda_{j}}{a} U_{8,5, j}^{m}+X_{j} U_{8,5, j}^{\prime m}+\frac{E_{1,5, j}^{m}}{a}+2 E_{1,5, j}^{\prime m} \\
& Z_{2,5, j}^{m}=\frac{\Lambda_{j}}{a} U_{9,5, j}^{m}+X_{j} U_{9,5, j}^{\prime m}+\frac{Z_{1,5, j}^{m}}{a}+2 Z_{1,5, j}^{\prime m}-T_{j} \frac{p}{\xi_{j}} \frac{J_{m}\left(\xi_{j} a\right)}{\xi_{j}}
\end{aligned}
$$

2.3.6. Projection on $-i \hat{\mathbf{z}} \mathbf{C}_{m}$

$$
\begin{aligned}
& A_{2,6, j}^{m}=\frac{\Lambda_{j}^{\prime}}{a} U_{1,6, j}^{m}+X_{j}^{\prime} U_{1,6, j}^{\prime m}+\frac{A_{1,6, j}^{m}}{a}+2 A_{1,6, j}^{\prime m} \\
& +\frac{1}{2}\left[4 \alpha_{1}+2 \alpha_{2}+3 \alpha_{3}\right] k_{j}^{2} \frac{p^{2}}{\xi_{j}^{2}} \frac{J_{m}\left(\xi_{j} a\right)}{a}, j=1,2
\end{aligned}
$$




$$
\begin{aligned}
& B_{2,6, j}^{m}=\frac{\Lambda_{j}^{\prime}}{a} U_{2,6, j}^{m}+X_{j}^{\prime} U_{2,6, j}^{\prime m}+\frac{B_{1,6, j}^{m}}{a}+2 B_{1,6, j}^{\prime m}, j=1,2 \\
& C_{2,6, j}^{m}=\frac{\Lambda_{j}^{\prime}}{a} U_{3,6, j}^{m}+X_{j}^{\prime} U_{3,6, j}^{\prime m}+\frac{C_{1,6, j}^{m}}{a}+2 C_{1,6, j}^{\prime m} \\
& +\frac{1}{2}\left[4 \alpha_{1}+2 \alpha_{2}+3 \alpha_{3}\right] k_{j}^{2} \frac{p^{2}}{\xi_{j}^{2}} \frac{J_{m}\left(\xi_{j} a\right)}{a} \\
& A_{2,6, j}^{m}=\frac{\Lambda_{j}}{a} U_{4,6, j}^{m}+X_{j} U_{4,6, j}^{\prime m}+\frac{A_{1,6, j}^{m}}{a}+2 A_{1,6, j}^{\prime m}-T_{j} \frac{\dot{J}_{m}\left(\xi_{j} a\right)}{\xi_{j}} \\
& -\frac{1}{2}\left[\alpha_{1}+\alpha_{3}\right] k_{j}^{2} \frac{J_{m}\left(\xi_{j} a\right)}{a}, j=3,4 \\
& B_{2,6, j}^{m}=\frac{\Lambda_{j}}{a} U_{5,6, j}^{m}+X_{j} U_{5,6, j}^{\prime m} \\
& +\frac{B_{1,6, j}^{m}}{a}+2 B_{1,6, j}^{\prime m}-T_{j} i m \frac{J_{m}\left(\xi_{j} a\right)}{\xi_{j}^{2} a}, j=3,4 \\
& \Gamma_{2,6, j}^{m}=\frac{\Lambda_{j}}{a} U_{6,6, j}^{m}+X_{j} U_{6,6, j}^{\prime m}+\frac{\Gamma_{1,6, j}^{m}}{a}+2 \Gamma_{1,6, j}^{\prime m}+T_{j} \frac{p^{2}}{\xi_{j}^{2}} \frac{\dot{J}_{m}\left(\xi_{j} a\right)}{\xi_{j}} \\
& +\frac{1}{2}\left[\alpha_{1}+\alpha_{3}\right] k_{j}^{2} \frac{p^{2}}{\xi_{j}^{2}} \frac{J_{m}\left(\xi_{j} a\right)}{a} \\
& \Delta_{2,6, j}^{m}=\frac{\Lambda_{j}}{a} U_{7,6, j}^{m}+X_{j} U_{7,6, j}^{\prime m}+\frac{\Delta_{1,6, j}^{m}}{a}+2 \Delta_{1,6, j}^{\prime m} \\
& E_{2,6, j}^{m}=\frac{\Lambda_{j}}{a} U_{8,6, j}^{m}+X_{j} U_{8,6, j}^{\prime m}+\frac{E_{1,6, j}^{m}}{a}+2 E_{1,6, j}^{\prime m} \\
& Z_{2,6, j}^{m}=\frac{\Lambda_{j}}{a} U_{9,6, j}^{m}+X_{j} U_{9,6, j}^{\prime m}+\frac{Z_{1,6, j}^{m}}{a}+2 Z_{1,6, j}^{\prime m}
\end{aligned}
$$

2.3.7. Projection on $\left(\hat{\mathbf{r}} \mathbf{B}_{m}\right)_{\alpha}$

$$
\begin{gathered}
A_{2,7, j}^{m}=\frac{\Lambda_{j}^{\prime}}{a} U_{1,7, j}^{m}+X_{j}^{\prime} U_{1,7, j}^{\prime m}+\frac{A_{1,7, j}^{m}}{a}+2 A_{1,7, j}^{\prime m}+Q_{j} i m \frac{J_{m}\left(\xi_{j} a\right)}{\xi_{j}^{2} a}, j=1(\mathbf{\text { . }} 149) \\
\left.B_{2,7, j}^{m}=\frac{\Lambda_{j}^{\prime}}{a} U_{2,7, j}^{m}+X_{j}^{\prime} U_{2,7, j}^{\prime m}+\frac{B_{1,7, j}^{m}}{a}+2 B_{1,7, j}^{\prime m}-Q_{j} \frac{\dot{J}_{m}\left(\xi_{j} a\right)}{\xi_{j}}, j=1, £ \mathrm{~A} .150\right) \\
C_{2,7, j}^{m}=\frac{\Lambda_{j}^{\prime}}{a} U_{3,7, j}^{m}+X_{j}^{\prime} U_{3,7, j}^{\prime m}+\frac{C_{1,7, j}^{m}}{a}+2 C_{1,7, j}^{\prime m}-Q_{j} \frac{p^{2}}{\xi_{j}^{2}} i m \frac{J_{m}\left(\xi_{j} a\right)}{\xi_{j}^{2} a} \text { (A.151) }
\end{gathered}
$$




$$
\begin{gathered}
A_{2,7, j}^{m}=\frac{\Lambda_{j}}{a} U_{4,7, j}^{m}+X_{j} U_{4,7, j}^{\prime m}+\frac{A_{1,7, j}^{m}}{a}+2 A_{1,7, j}^{\prime m}-T_{j} i m \frac{J_{m}\left(\xi_{j} a\right)}{\xi_{j}^{2} a}, j=3,(\mathbb{A} .152) \\
B_{2,7, j}^{m}=\frac{\Lambda_{j}}{a} U_{5,7, j}^{m}+X_{j} U_{5,7, j}^{\prime m}+\frac{B_{1,7, j}^{m}}{a}+2 B_{1,7, j}^{\prime m}+T_{j} \frac{\dot{J}_{m}\left(\xi_{j} a\right)}{\xi_{j}}, j=3,4 \text { (A.153) } \\
\Gamma_{2,7, j}^{m}=\frac{\Lambda_{j}}{a} U_{6,7, j}^{m}+X_{j} U_{6,7, j}^{\prime m}+\frac{\Gamma_{1,7, j}^{m}}{a}+2 \Gamma_{1,7, j}^{\prime m}+T_{j} i m \frac{p^{2}}{\xi_{j}^{2}} \frac{J_{m}\left(\xi_{j} a\right)}{\xi_{j}^{2} a} \quad \text { (A.154) } \\
\Delta_{2,7, j}^{m}=\frac{\Lambda_{j}}{a} U_{7,7, j}^{m}+X_{j} U_{7,7, j}^{\prime m}+\frac{\Delta_{1,7, j}^{m}}{a}+2 \Delta_{1,7, j}^{\prime}+T_{j} \frac{\dot{J}_{m}\left(\xi_{j} a\right)}{\xi_{j}} \quad \text { (A.155) } \\
E_{2,7, j}^{m}=\frac{\Lambda_{j}}{a} U_{8,7, j}^{m}+X_{j} U_{8,7, j}^{\prime m}+\frac{E_{1,7, j}^{m}}{a}+2 E_{1,7, j}^{\prime m}+T_{j} i m \frac{J_{m}\left(\xi_{j} a\right)}{\xi_{j}^{2} a} \quad \text { (A.156) } \\
Z_{2,7, j}^{m}=\frac{\Lambda_{j}}{a} U_{9,7, j}^{m}+X_{j} U_{9,7, j}^{\prime m}+\frac{Z_{1,7, j}^{m}}{a}+2 Z_{1,7, j}^{\prime m}-T_{j} \frac{p^{2}}{\xi_{j}^{2}} \frac{\dot{J}_{m}\left(\xi_{j} a\right)}{\xi_{j}} \quad \text { (A.157) }
\end{gathered}
$$

2.3.8. Projection on $\left(\hat{\mathbf{r}} \mathbf{C}_{m}\right)_{\alpha}$

$$
\begin{gathered}
A_{2,8, j}^{m}=\frac{\Lambda_{j}^{\prime}}{a} U_{1,8, j}^{m}+X_{j}^{\prime} U_{1,8, j}^{\prime m}+\frac{A_{1,8, j}^{m}}{a}+2 A_{1,8, j}^{\prime m} \\
+Q_{j} \frac{p}{\xi_{j}} \frac{J_{m}\left(\xi_{j} a\right)}{\xi_{j}}+\frac{1}{2}\left[4 \alpha_{1}+2 \alpha_{2}+3 \alpha_{3}\right] k_{j}^{2} \frac{p}{\xi_{j}} \frac{\dot{J}_{m}\left(\xi_{j} a\right)}{a}, j=1,2(\mathrm{~A} .158) \\
B_{2,8, j}^{m}=\frac{\Lambda_{j}^{\prime}}{a} U_{2,8, j}^{m}+X_{j}^{\prime} U_{2,8, j}^{\prime m}+\frac{B_{1,8, j}^{m}}{a}+2 B_{1,8, j}^{\prime m} \\
+\frac{1}{2}\left[4 \alpha_{1}+2 \alpha_{2}+3 \alpha_{3}\right] k_{j}^{2} i m \frac{p}{\xi_{j}} \frac{J_{m}\left(\xi_{j} a\right)}{\xi_{j} a^{2}}, j=1,2 \\
C_{2,8, j}^{m}=\frac{\Lambda_{j}^{\prime}}{a} U_{3,8, j}^{m}+X_{j}^{\prime} U_{3,8, j}^{\prime}+\frac{C_{1,8, j}^{m}}{a}+2 C_{1,8, j}^{\prime m} \\
+Q_{j} \frac{p}{\xi_{j}} \frac{J_{m}\left(\xi_{j} a\right)}{\xi_{j}}-\frac{1}{2}\left[4 \alpha_{1}+2 \alpha_{2}+3 \alpha_{3}\right] k_{j}^{2} \frac{p^{3}}{\xi_{j}^{3}} \frac{\dot{j}_{m}\left(\xi_{j} a\right)}{a} \\
A_{2,8, j}^{m}=\frac{\Lambda_{j}}{a} U_{4,8, j}^{m}+X_{j} U_{4,8, j}^{\prime m}+\frac{A_{1,8, j}^{m}}{a}+2 A_{1,8, j}^{\prime m}-T_{j} \frac{p}{\xi_{j}} \frac{J_{m}\left(\xi_{j} a\right)}{\xi_{j}} \\
\left.+\frac{1}{2}\left[\alpha_{1}+\alpha_{3}+2 \alpha_{4}+3 \alpha_{5}\right] k_{j}^{2} \frac{p}{\xi_{j}} \frac{\dot{J}_{m}\left(\xi_{j} a\right)}{a}, j=3,400\right)
\end{gathered}
$$




$$
\begin{gathered}
B_{2,8, j}^{m}=\frac{\Lambda_{j}}{a} U_{5,8, j}^{m}+X_{j} U_{5,8, j}^{\prime m}+\frac{B_{1,8, j}^{m}}{a}+2 B_{1,8, j}^{\prime m} \\
+\frac{1}{2}\left[2 \alpha_{4}+3 \alpha_{5}\right] k_{j}^{2} i m \frac{p}{\xi_{j}} \frac{J_{m}\left(\xi_{j} a\right)}{\xi_{j} a^{2}}, j=3,4 \\
\Gamma_{2,8, j}^{m}=\frac{\Lambda_{j}}{a} U_{6,8, j}^{m}+X_{j} U_{6,8, j}^{\prime m}+\frac{\Gamma_{1,8, j}^{m}}{a}+2 \Gamma_{1,8, j}^{\prime m}-T_{j} \frac{p}{\xi_{j}} \frac{J_{m}\left(\xi_{j} a\right)}{\xi_{j}} \\
+\frac{1}{2}\left[\alpha_{1}+\alpha_{3}\right] k_{j}^{2} \frac{p}{\xi_{j}} \frac{\dot{J}_{m}\left(\xi_{j} a\right)}{a}-\frac{1}{2}\left[2 \alpha_{4}+3 \alpha_{5}\right] k_{j}^{2} \frac{p^{3}}{\xi_{j}^{3}} \frac{\dot{J}_{m}\left(\xi_{j} a\right)}{a} \\
\Delta_{2,8, j}^{m}=\frac{\Lambda_{j}}{a} U_{7,8, j}^{m}+X_{j} U_{7,8, j}^{\prime m}+\frac{\Delta_{1,8, j}^{m}}{a}+2 \Delta_{1,8, j}^{\prime m} \\
E_{2,8, j}^{m}=\frac{\Lambda_{j}}{a} U_{8,8, j}^{m}+X_{j} U_{8,8, j}^{\prime m}+\frac{E_{1,8, j}^{m}}{a}+2 E_{1,8, j}^{\prime m} \\
Z_{2,8, j}^{m}=\frac{\Lambda_{j}}{a} U_{9,8, j}^{m}+X_{j} U_{9,8, j}^{\prime m}+\frac{Z_{1,8, j}^{m}}{a}+2 Z_{1,8, j}^{\prime m}
\end{gathered}
$$

2.3.9. Projection on $\left(\hat{\phi} \mathbf{C}_{m}\right)_{\alpha}$

$$
\begin{gathered}
A_{2,9, j}^{m}=\frac{\Lambda_{j}^{\prime}}{a} U_{1,9, j}^{m}+X_{j}^{\prime} U_{1,9, j}^{\prime m}+\frac{A_{1,9, j}^{m}}{a}+2 A_{1,9, j}^{\prime m} \\
+\frac{1}{2}\left[4 \alpha_{1}+2 \alpha_{2}+3 \alpha_{3}\right] k_{j}^{2} i m \frac{p}{\xi_{j}} \frac{J_{m}\left(\xi_{j} a\right)}{\xi_{j} a^{2}}, j=1,2 \\
B_{2,9, j}^{m}=\frac{\Lambda_{j}^{\prime}}{a} U_{2,9, j}^{m}+X_{j}^{\prime} U_{2,9, j}^{\prime m}+\frac{B_{1,9, j}^{m}}{a}+2 B_{1,9, j}^{\prime m} \\
-\frac{1}{2}\left[4 \alpha_{1}+2 \alpha_{2}+3 \alpha_{3}\right] k_{j}^{2} \frac{p}{\xi_{j}} \frac{\dot{J}_{m}\left(\xi_{j} a\right)}{a}, j=1,2 \\
C_{2,9, j}^{m}=\frac{\Lambda_{j}^{\prime}}{a} U_{3,9, j}^{m}+X_{j}^{\prime} U_{3,9, j}^{\prime m}+\frac{C_{1,9, j}^{m}}{a}+2 C_{1,9, j}^{\prime m} \\
-\frac{1}{2}\left[4 \alpha_{1}+2 \alpha_{2}+3 \alpha_{3}\right] k_{j}^{2} i m \frac{p^{3}}{\xi_{j}^{3}} \frac{J_{m}\left(\xi_{j} a\right)}{\xi_{j} a^{2}} \\
A_{2,9, j}^{m}=\frac{\Lambda_{j}}{a} U_{4,9, j}^{m}+X_{j} U_{4,9, j}^{\prime m}+\frac{A_{1,9, j}^{m}}{a}+2 A_{1,9, j}^{\prime m} \\
+\frac{1}{2}\left[2 \alpha_{4}+3 \alpha_{5}+\alpha_{1}+\alpha_{3}\right] k_{j}^{2} i m \frac{p}{\xi_{j}} \frac{J_{m}\left(\xi_{j} a\right)}{\xi_{j} a^{2}}, j=3,4
\end{gathered}
$$




$$
\begin{gathered}
B_{2,9, j}^{m}=\frac{\Lambda_{j}}{a} U_{5,9, j}^{m}+X_{j} U_{5,9, j}^{\prime m}+\frac{B_{1,9, j}^{m}}{a}+2 B_{1,9, j}^{\prime m} \\
-\frac{1}{2}\left[2 \alpha_{4}+3 \alpha_{5}\right] k_{j}^{2} \frac{p}{\xi_{j}} \frac{\dot{J}_{m}\left(\xi_{j} a\right)}{a}, j=3,4 \\
\Gamma_{2,9, j}^{m}=\frac{\Lambda_{j}}{a} U_{6,9, j}^{m}+X_{j} U_{6,9, j}^{\prime m}+\frac{\Gamma_{1,9, j}^{m}}{a}+2 \Gamma_{1,9, j}^{\prime m} \\
+\frac{1}{2}\left[\alpha_{1}+\alpha_{3}\right] k_{j}^{2} \frac{p}{\xi_{j}} i m \frac{J_{m}\left(\xi_{j} a\right)}{\xi_{j} a^{2}}-\frac{1}{2}\left[2 \alpha_{4}+3 \alpha_{5}\right] k_{j}^{2} \frac{p^{3}}{\xi_{j}^{3}} i m \frac{J_{m}\left(\xi_{j} a\right)}{\xi_{j} a^{2}}(\mathrm{~A} .1 \mathrm{~A} \\
\Delta_{2,9, j}^{m}=\frac{\Lambda_{j}}{a} U_{7,9, j}^{m}+X_{j} U_{7,9, j}^{\prime m}+\frac{\Delta_{1,9, j}^{m}}{a}+2 \Delta_{1,9, j}^{\prime m}-T_{j} \frac{p}{\xi_{j}} \frac{J_{m}\left(\xi_{j} a\right)}{\xi_{j}} \\
E_{2,9, j}^{m}=\frac{\Lambda_{j}}{a} U_{8,9, j}^{m}+X_{j} U_{8,9, j}^{\prime m}+\frac{E_{1,9, j}^{m}}{a}+2 E_{1,9, j}^{\prime m} \\
Z_{2,9, j}^{m}=\frac{\Lambda_{j}}{a} U_{9,9, j}^{m}+X_{j} U_{9,9, j}^{\prime m}+\frac{Z_{1,9, j}^{m}}{a}+2 Z_{1,9, j}^{\prime m}-T_{j} \frac{p}{\xi_{j}} \frac{J_{m}\left(\xi_{j} a\right)}{\xi_{j}}
\end{gathered}
$$

\section{References}

[1] A. Charalambopoulos and L.N.Gergidis On the Dyadic Scattering Problem in 3-Dimensional Gradient Elasticity: Analytic Approach Journal of Physics A, Mathematical and Theoretical,41(39),Article Number: 395203,2008

[2] R.D. Mindlin. Microstructures in linear elasticity. Arch. Rat. Mech. Anal., 10:51-78, 1964.

[3] R.D. Mindlin. Second gradient of strain and surface tension in linear elasticity. Int.J.Solids Struct., 1:417-438, 1965.

[4] R.D. Mindlin and N.N.Eshel. On first strain-gradient theories in linear elasticity. Int.J.Solids Struct., 4:109-124, 1968.

[5] E.C. Aifantis. On the role of gradients in the localization of deformation and fracture. Int.J.Eng.Sci., 30:1279-1299, 1992.

[6] S. Altan and E.C. Aifantis. On the structure of the mode iii crack-tip in gradient elasticity. Scripta Metall. Mater., 26:319-324, 1992.

[7] C.Q. Ru and E.C. Aifantis. A simple approach to solve boundary value problems in gradient elasticity. Acta Mech., 101:59-68, 1993.

[8] E.C. Aifantis. Update on a class of gradient theories. Mech Matls., 35:259280, 2003. 
[9] I. Vardoulakis and J. Sulem. Bifurcation Analysis in Geomechanics . Blackie/Chapman and Hall, London, 1995.

[10] G.E. Exadaktylos and I.Vardoulakis. Microstructure in linear elasticity and scale effects: a reconsideration of basic rock mechanics and rock fracture mechanics. Tectonophysics, 335:81-109, 2001.

[11] A.C. Eringen and E.S. Suhubi. Nonlinear theory of simple micro-elastic solids. Int.J.Eng.Sci., 2:189-203, 1964.

[12] A.C. Eringen. Theory of micropolar elasticity. in:Liebowitz(Ed.),Fracture, Academic Press, New York, II:189-203, 1968.

[13] H. Kadowaki and W.K Liu. A multiscale approach for the micropolar continuum model. CMES: Computer Modeling in Engineering \& Sciences, 7:269-282, 2005.

[14] E.Cosserat and F.Cosserat Theorie des Corps Deformables. A.Hermann et Cie,Paris, 1909.

[15] R.D. Mindlin and H.F. Tiersten. Effects of couple-stresses in linear elasticity. Arch. Rat. Mech. Anal., 11:415-448, 1962.

[16] W.T. Koiter. Theories of elasticity with couple-stresses. I II.,Proc.K.Ned.Akad.Wet.B, 67:17-44, 1964.

[17] R.A. Toupin. Theories of elasticity with couple-stresses. Arch. Rat. Mech. Anal., 17:85-112, 1965.

[18] K.G. Tsepoura, S. Papargyri-Beskou, D. Polyzos, and D.E. Beskos. Static and dynamic analysis of a gradient elastic bar in tension. Arch. Appl. Mech., 72:483-497, 2002.

[19] H.G. Georgiadis, I. Vardoulakis, and G. Lykotrafitis. Torsional surface waves in a gradient-elastic half-space. Wave Motion, 31:333-348, 2000.

[20] L.F. Qian, R.C. Batra, and L.M. Chen. Elastostatic Deformations of a Thick Plate by using a Higher -Order Shear and Normal Deformable Plate Theory and two Meshless Local Petrov-Galerkin (MLPG) Methods. CMES: Computer Modeling in Engineering \& Sciences, 4:161-176, 2003.

[21] H.G. Georgiadis and E.G. Velgaki. High-frequency rayleigh waves in materials with micro-structure and couple-stress effects. International Journal of Solids and Structures, 40:2501-2520, 2003.

[22] H.G. Georgiadis, I.Vardoulakis, and E.G. Velgaki. Dispersive rayleigh-wave propagation in microstructured solids characterized by dipolar gradient elasticity. Journal of Elasticity, 74:17-45, 2004. 
[23] M. Vavva, V. Protopappas, L.N.Gergidis, A. Charalambopoulos, D. Fotiadis and D. Polyzos The effect of boundary conditions on guided wave propagation in 2-dimensional models of healing bone Ultrasonics,48(6/7): $598-606,2008$

[24] M. Vavva, V. Protopappas, L.N.Gergidis, A. Charalambopoulos, D.I. Fotiadis and D. Polyzos Velocity Dispersion Curves of Guided Waves Propagating in a Free Gradient Elastic Plate: Application to Cortical Bone Journal of the Acoustical Society of America (JASA),125(5), 3414-3427,2009

[25] A. Charalambopoulos, D.I. Fotiadis, C.V. Massalas, Free vibrations of a double layered elastic isotropic cylindrical rod International Journal of Engineering Sciences, 36, 711-731, 1998

[26] A. Charalambopoulos, D.I. Fotiadis, C.V. Massalas, Free Vibrations of the Viscoelastic Human Skull, International Journal of Engineering Sciences, $36(5 / 6)$, pp. 565-576, 1998

[27] A. Charalambopoulos, G.Dassios, D.I. Fotiadis, C.V. Massalas, Dynamic Characteristics of the Human Skull-Brain System, Math. Comput. Modelling, 27 (2), pp. 81-101, 1998

[28] G. Dassios, K. Kiriaki, and D.Polyzos, Scattering theorems for complete dyadic fields. International Journal of Engineering Sciences, 33:269277, 1995.

[29] A.Charalambopoulos, G.Dassios,D.I.Fotiadis, C.V.Massalas Note on eigenvector solutions of the Navier equation in cylindrical coordinates Acta Mechanica, 134(1/2),pp.115-119, 1999.

[30] Z. Tang, S. Shen, and S.N. Atluri. Analysis of Materials with StrainGradient Effects: A Meshless Local Petrov-Galerkin(MLPG) Approach with Nodal Displacements only. CMES: Computer Modeling in Engineering 83 Sciences, 4:177-196, 2003.

[31] J. Agnantiaris and D. Polyzos. A boundary element method for acoustic scattering from non-axisymmetric and axisymmetric elastic shells . CMES: Computer Modeling in Engineering \& Sciences, 4:197-212, 2003.

[32] P. Santos, J. Antonio, and A. Tadeu. Wave Scattering by 2D Smooth Topographical Elastic Deformations Caused by a Point Blast Source. CMES: Computer Modeling in Engineering ES Sciences, 1:79-97, 2000.

[33] C. Eringen. Microcontinuum Field Theories I: Foundations and Solids. Springer, 1999.

[34] G. Dassios, K. Kiriaki, and D.Polyzos. Scattering theorems for complete dyadic fields. International Journal of Engineering Sciences, 33:269-277, 1995. 
[35] D. Polyzos, K.G. Tsepoura, S.V.Tsinopoulos, and D.E. Beskos. A boundary element method for solving 2-d and 3-d static gradient elastic problems.Part I: integral formulation. Comput.Methods Appl.Mech.Engrg, 192:2845-2873, 2003.

[36] G. Dassios and I.V. Lindell. On the Helmholtz decomposition for polydyadics. Quarterly of Applied Mathematics, 59(4):787-796, 2001.

[37] P. Morse and H. Feshbach. Methods of Theoretical Physics. McGraw-Hill, New York, 1953.

[38] A. Papacharalampopoulos, M. Vavva, V. Protopappas, D.I. Fotiadis and D. Polyzos A numerical study on the propagation of Rayleigh and guided waves in cortical bone according to Mindlin's Form II gradient elastic theory. Journal of the Acoustical Society of America (JASA),130(2), 1060-1070,2011

[39] V. Protopappas, I.C. Kourtis, L.C. Courtis, K.N.Malizos, C.V. Massalas, D.I. Fotiadis Three dimensional finite element modeling of guided ultrasound wave propagation in intact and healing long bone. Journal of the Acoustical Society of America (JASA),121(6), 3907-3921,2007

[40] A.C. Eringen. Linear theory of micropolar elasticity. J. Math. Mech. 15,909923, 1966.

[41] A.C.Eringen. Nonlocal Continuum Field Theories. Springer,New York, 2002

[42] M. Ben Amoz. A dynamic theory for composite materials. J. Appl. Math. Phys.,27, 83-99,1976 